\title{
Numerical investigation to predict optimum attack angle combination of longitudinal vortex generators in compact heat exchangers for thermo-hydraulic heightened performance
}

\author{
MOHD ZEESHAN, SUJIT NATH*(1) and DIPANKAR BHANJA \\ Department of Mechanical Engineering, National Institute of Technology Silchar, Silchar 788010, India \\ e-mail: sujitnath2008@gmail.com
}

MS received 10 April 2019; revised 11 September 2019; accepted 13 September 2019

\begin{abstract}
The current 3-D numerical analysis explores the effect of combinations of rectangular winglet pairs (RWPs) having different attack angles (i.e. $5^{\circ}, 15^{\circ}$ and $25^{\circ}$ ) along a row of the tube array, on the performance of the fin and tube heat exchanger (FTHE). The considered airside Reynolds number Re ranges from 500 to 900 . In total, six combinations of three attack angle vortex generators (VGs) have been numerically analysed namely $5^{\circ}$ $15^{\circ}-25^{\circ}, 5^{\circ}-25^{\circ}-15^{\circ}, 15^{\circ}-5^{\circ}-25^{\circ}, 15^{\circ}-25^{\circ}-5^{\circ}, 25^{\circ}-5^{\circ}-15^{\circ}$ and $25^{\circ}-15^{\circ}-5^{\circ}$. The performance of the FTHE is represented by area goodness factor. The performance rankings of the FTHEs are also obtained by the MOORA method. Finally, $5^{\circ}-25^{\circ}-15^{\circ}$ case provides the best thermal hydraulic performance for which heat transfer coefficient $(h)$ is increased by $68.20 \%$ at $R e=500$ and $81.78 \%$ at $R e=900$, with a significant pressure drop penalty.
\end{abstract}

Keywords. Fin and tube heat exchanger; rectangular vortex generators; area goodness factor; MOORA method.

\section{Introduction}

Augmentation in the performance of compact heat exchangers is becoming more popular among the researchers as these heat exchangers have a widespread use; e.g. refrigeration, power generation, car radiators, HVAC systems and so forth. Vortex generators (VGs) promise the augmented performance of heat exchangers (HXs) by establishing secondary flow in the flow domain. An extensive review of the vortex generators implementation for enhancing the thermal performance has been presented by Jacobi and Shah [1]. The review describes the potential of VGs in heat transfer enhancement and provides a base for further research work. Bendaoud et al [2] proposed a novel modelling approach of finned tube heat exchangers (FTHEs) for the performance evaluation. Incorporation of VGs with enhanced surfaces (louvered, wavy and slit fins) and modified tubes (flat and oval), serves better thermal performance of heat exchangers [3-5]. Tiwari et al [6] investigated the use of oval tubes and multiple delta VGs to obtain the enhanced thermal-hydraulic performance of compact heat exchangers. The thermal performance in terms of Nusselt number $(\mathrm{Nu})$ was increased by $100 \%$ in case of oval tube and multiple delta VGs, compared to the baseline case in which no VGs were used. Experimental

*For correspondence

Published online: 22 November 2019 investigation performed by Leu et al [7] explored the effect of using rectangular blocks as VGs behind the tubes. The fin area can be reduced up to $25 \%$ by the incorporation of proposed configuration of VGs at $R e=500$. Joardar and Jacobi [8] experimentally investigated the optimum attack angle of delta VGs to obtain the augmented thermal-hydraulic performance of FTHEs. An enhancement of 29.9-68.8\% in the heat transfer coefficient $(h)$ was achieved for three row VGs array arrangement projected in the study. Kumar et al [9] presented a review on the performance characteristics of the air-cooled Heat exchangers which provide the scope of further research in performance enhancement of the FTHEs. Arshad et al [10] experimentally explored the fin geometry effect on the flow induced vibration in finned tube HXs. It was found in the study that the tube experiences maximum vibrations in the third row.

Several numerical studies also carried out to perceive the thermal hydraulic behaviour on the implementation of VGs. $\mathrm{Wu}$ and Tao [11] investigated that the longitudinal vortices can improve the synergy between velocity and temperature field in the region near LVG (longitudinal vortex generators) and in the large downstream region of LVG whereas transverse vortices have the significant effect on the synergy in the region near LVG. Lin et al [12] investigated that an improved thermal performance can be achieved by the heat exchanger with oval tubes and inclined block shape VGs. He et al [13] performed numerical analysis with various arrangements of 
winglets and found that front continuous winglet arrays provide the maximum value of area goodness factor $(j / f)$. He et al [14] also carried out a numerical study on rectangular winglet VGs and found that altering the placement of the same number of rectangular vortex generators (RWPs) from inline array to staggered array reduces the pressure loss penalty without decreasing the heat transfer. A significant increment in the heat transfer coefficient $(h)$ was found i.e., $28.8-34.5 \%, 54.6-61.5 \%$ and $83.3-89.7 \%$ when attack angles are taken as $10^{\circ}, 20^{\circ}$ and $30^{\circ}$, respectively. Zeeshan et al $[15,16]$ optimized the angle of attack of RWPs oriented in CFD (common flow down) and CFU (common flow up) manner at different locations by using MOORA method to get improved heat transfer at the expense of fan power consumption, high area goodness factor and low fan power consumption. A numerical study performed by Sinha et al [17] demonstrated that in staggered arrangement of the tubes, and with alternate CFD-CFU orientations of the VGs, increase in angle of attack up to a certain point, results an augmentation in thermal performance but further increment in attack angle reduces the performance. Sharma et al [18] found a significant effect of variation in the winglet thickness on the performance of plate fin heat exchanger. It was found in the study that adding thickness leads to the increment of $19.7 \%$ in the heat transfer coefficient $(h)$ with a marginal pressure drop penalty of $7.8 \%$. Sarangi and Mishra [19] numerically investigated the steam-wise and span-wise locations to get higher thermal hydraulic performances. Again, apart from the rectangular and delta winglet type VGs some non-traditional VGs also studied and compared with traditional VGs. Lin et al [20] proposed fins, embedded with wave type vortex generators and predicted an $18-20 \%$ fin area reduction by implementation of enhanced fins. Semidimples, full dimples [21, 22] wavy up, wavy down [23], inclined projected winglet pair (IPWP) [24] and curved winglets $[25,26]$ are some non-conventional type VGs which have been implemented to get higher thermal hydraulic performance.

In the past literatures [14-17, 19], authors kept constant attack angles for RWPs. It can be concluded from the past literature survey that higher attack angle of RWPs provides the higher thermal performance but with a significant pressure drop penalty. It is interesting to find the effect of placement of RWPs having different attack angles, on the thermal hydraulic performances of a FTHE. This study provides a comprehensive analysis of thermal hydraulic performances of a FTHE with RWPs having different attack angles placed adjacent to the tubes in common flow up manner. So that readers can visualize and understand the thermal and hydraulic characteristics well. Moreover, in the previous work [14-17] the effect of placement of RWPs with constant attack angle on the performance of FTHEs has already been reported. As it is the extension of the previous works, it will provide significant information for the compact heat exchanger design and optimization. In the present work three VGs of different attack angle are mounted adjacent to $1^{\text {st }}, 3^{\text {rd }}$ and $5^{\text {th }}$ tube. The numerical simulations have been run in the steady state condition. Moreover, a total number of six combinations of different attack angles of vortex generators have been analysed extensively in this study. Performance evaluation of finned tube heat exchangers has been done by area goodness factor $(j / f)$ and heat transfer rate per unit fan power consumption $\left(Q / P_{f}\right)$. Moreover, MOORA method $[16,27]$ is also applied in this study to obtain the order of performance of finned tube heat exchangers having different combinations of VGs. The sole objective of MOORA in this study is to obtain the configuration having high thermal performance $(\mathrm{Nu})$, high compactness $(\mathrm{j} / \mathrm{f})$ and with a reduced friction factor $(f)$. That is Nusselt number $(\mathrm{Nu})$ and area goodness factor $(j / f)$ are considered as beneficial attributes (to be maximized) and friction factor $(f)$ is considered as a nonbeneficial attribute (to be minimized).

\section{Description of the model}

\subsection{Physical model}

In this numerical study RWPs are attached on the fin surface symmetrically in CFU orientation adjacent to the tube numbers 1,3 and 5 as shown in figures 1 and 2 . The geometrical description of the considered computational domain in $\mathrm{x}-\mathrm{y}$ plane (shown by hatched lines), top view of the RWP and the side view of RWP, where dashed lines show the region of the considered computational domain in $\mathrm{x}-\mathrm{z}$ plane are shown in figures $1(\mathrm{a})$, (b) and (c), respectively. In total, six combinations of three attack angle VGs $\left(5^{\circ}-15^{\circ}-25^{\circ}, 5^{\circ}-25^{\circ}-15^{\circ}, 15^{\circ}-5^{\circ}-25^{\circ}, 1^{\circ}-25^{\circ}-5^{\circ}, 25^{\circ}-5^{\circ}\right.$ $15^{\circ}$ and $25^{\circ}-15^{\circ}-5^{\circ}$ ) have been numerically analysed after getting the optimised winglet height. The physical model of the interest in the current study has been taken from the experimental study performed by Joardar and Jacobi [8]. All the geometrical parameters are taken as same from the study [8], but this study considers rectangular VGs. The considered channel height is $3.63 \mathrm{~mm}$ and both longitudinal and transverse tube pitch have the same dimensions of $25.4 \mathrm{~mm}$ [28]. An extension of $5 \mathrm{H}$ is given at the inlet to obtain the uniformity of the inlet velocity and an extension of $30 \mathrm{H}$ is given at the outlet to maintain a recirculation-free flow $[14-16,27,28]$. Where $H$ is the channel height $(3.63 \mathrm{~mm})$ as shown in figure $1(\mathrm{c})$.

\subsection{Numerical procedure}

In the present numerical study, the fluid (air) is considered as incompressible and with constant properties. The present evaporator has the geometric character of symmetry and periodicity so only one eighth part of the total fin plate is modelled in Design Modeller ANSYS 14.0 as shown in figure 2(a). A hybrid type of the mesh has been generated in workbench ANSYS 14.0 (ICEM CFD). The multi-zone 


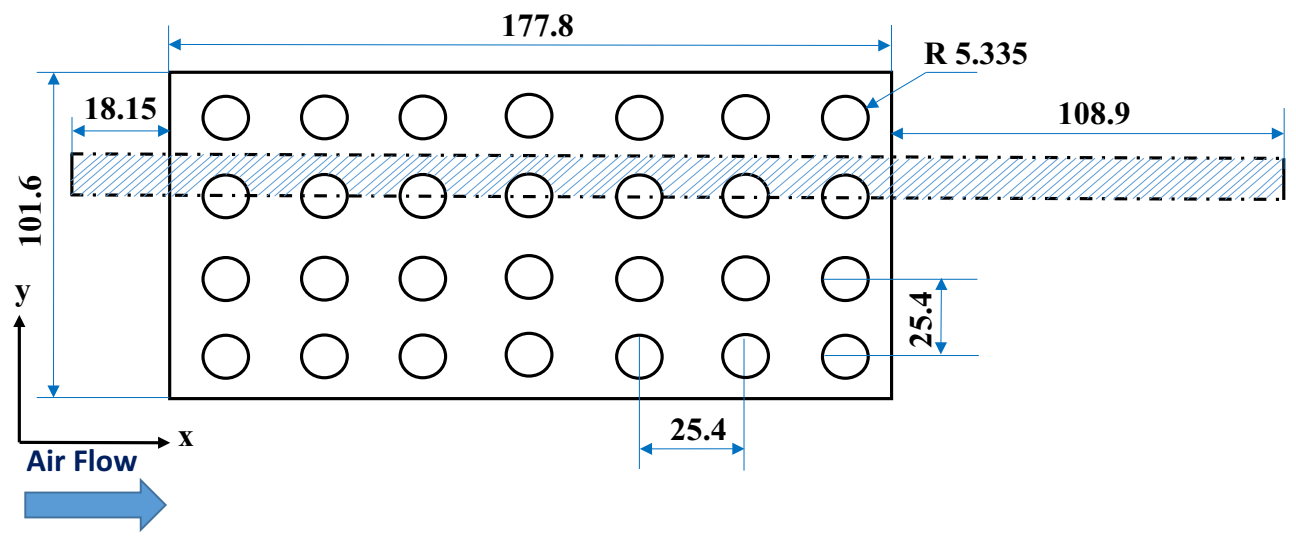

(a)

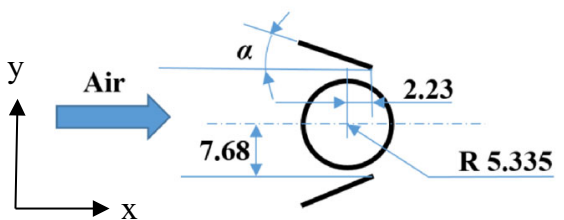

(b)

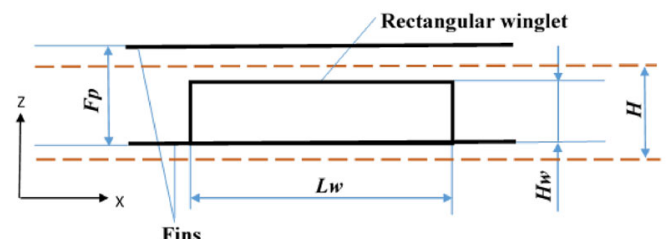

(c)

Figure 1. (a) Geometric description of considered computational domain (all dimensions are in mm); (b) top view of RWP; (c) side view of the RWP.

method has been adopted for the mesh generation and body sizing operation has been adopted with $0.3 \mathrm{~mm}$ size of the element (for the grid system having 599835 number of elements), to obtain the finer mesh in the computational domain. The structured mesh has been generated with hexahedral mesh at inlet and outlet extended zones whereas unstructured mesh has been generated around the tubes and rectangular winglets. Figure 2(b) depicts the generated mesh with description of the adopted meshing strategy in various regions of computational domain. FLUENT ANSYS 14.0 is used as a solver for current numerical problem. The second order upwind scheme has been employed to discretise the convective terms of the governing equations for momentum and energy conservation, and SIMPLE algorithm is used to solve pressure velocity coupled equation. The small fin pitch $(3.63 \mathrm{~mm})$ and lower inlet velocities having the range of $R e$ as 500-900 enables taking the flow as laminar and steady. The solution of the conjugate heat transfer problem in the computational domain provides the distribution of the temperature over the fins.

The governing equations can be expressed as follows $[15,17,27]$ :

$$
\begin{gathered}
\text { Continuity: } \quad \frac{\partial u_{i}}{\partial x_{i}}=0 \\
\text { Momentum: } \quad \frac{\partial\left(\rho u_{i} u_{k}\right)}{\partial x_{i}}=\frac{\partial}{\partial x_{i}}\left(\mu \frac{\partial u_{k}}{\partial x_{i}}\right)-\frac{\partial p}{\partial x_{k}} \\
\text { Energy: } \quad \frac{\partial\left(\rho u_{i} T\right)}{\partial x_{i}}=\frac{\partial}{\partial x_{i}}\left(\frac{\lambda}{c_{p}} \frac{\partial T}{\partial x_{i}}\right)
\end{gathered}
$$

The boundary conditions for the considered regions can be seen in figure 2(a) and also described as follows:

- At inlet boundary:

$$
u=u_{\text {in }}=\text { constant, } v=w=0, T=T_{\text {in }}=\text { constant }
$$

- At side boundaries of fluid regions:

$$
\frac{\partial u}{\partial y}=\frac{\partial w}{\partial y}=0, v=0, \frac{\partial T}{\partial y}=0
$$

- At side boundaries of fin surface: (solid surface)

$$
u=v=w=0, \frac{\partial T}{\partial y}=0
$$

- Tube surface:

$$
u=v=w=\text { constant, } T=T_{w}=\text { constant }
$$

- At the top and the bottom boundaries:

$$
\begin{gathered}
\text { Periodic condition for velocity } \quad u_{u p}=u_{\text {down }} \\
\text { Periodic condition for temperature } \quad T_{u p}=T_{\text {down }}
\end{gathered}
$$

- At outlet boundary:

$$
\frac{\partial u}{\partial x}=\frac{\partial v}{\partial x}=\frac{\partial w}{\partial x}=0, \frac{\partial T}{\partial x}=0
$$

\subsection{Parameter definitions}

The considered non-dimensional numbers and different parameters are defined as follows: 


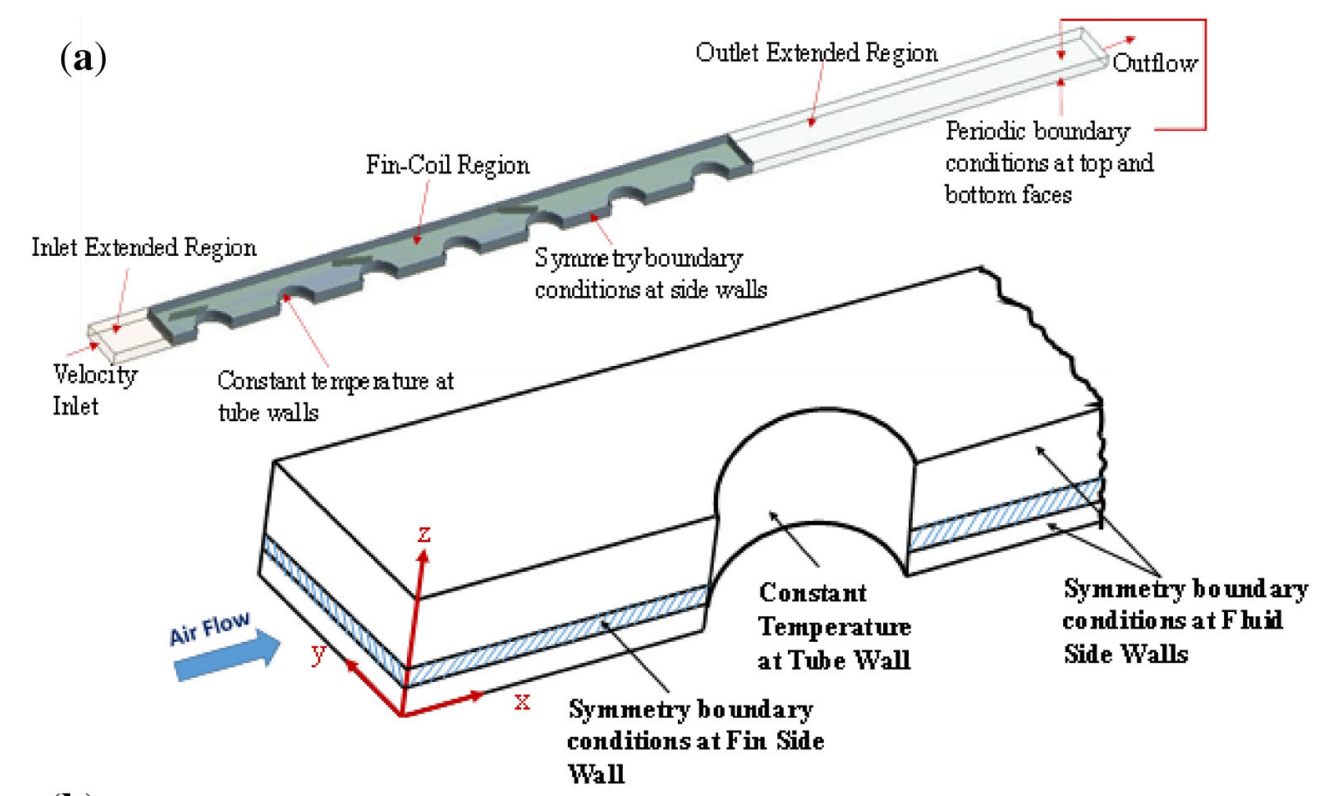

(b)

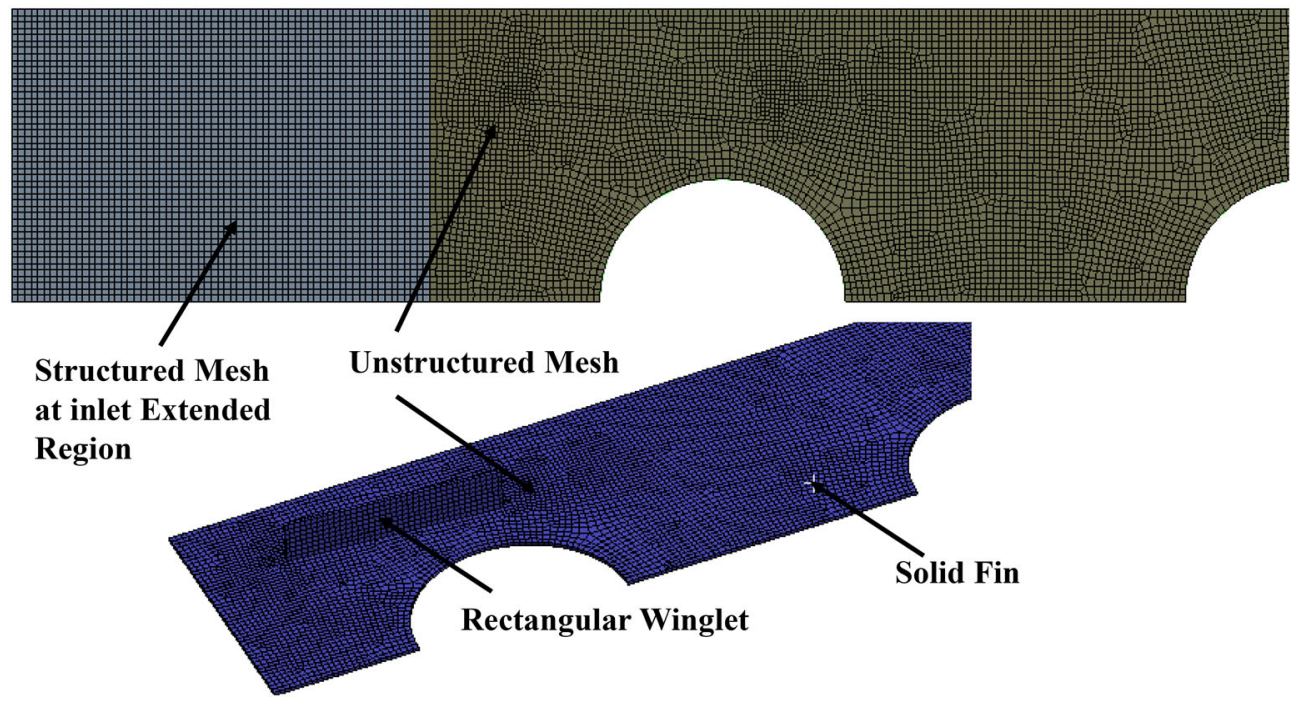

Figure 2. Representation of (a) three dimensional computational domain with necessary boundary conditions; (b) generated mesh at various zones of computational model.

$$
\begin{gathered}
\left\{\begin{array}{c}
\operatorname{Re} \\
D_{h} \\
N u \\
j \\
f
\end{array}\right\}=\left\{\begin{array}{c}
\left(\rho V_{m} D_{h}\right) / \mu \\
\left(4 A_{\min } L\right) / A_{T} \\
h D_{h} / k_{f} \\
S t . \operatorname{Pr}^{2 / 3} \\
\Delta p /\left(\frac{\rho V_{m}^{2}}{2} \frac{A_{T}}{A_{\min }}\right)
\end{array}\right\} ; \\
\left\{\begin{array}{c}
Q \\
\Delta p \\
\Delta T_{m} \\
h \\
\dot{m}_{p}\left(\bar{T}_{\text {out }}-\bar{T}_{\text {in }}\right) \\
\bar{p}_{\text {in }}-\bar{p}_{\text {out }} \\
P_{f}
\end{array}\right\}=\left\{\begin{array}{c}
\left(\bar{T}_{w}-\bar{T}_{\text {in }}\right)-\left(\bar{T}_{w}-\bar{T}_{\text {out }}\right) \\
\frac{\ln \left[\left(\bar{T}_{w}-\bar{T}_{\text {in }}\right) /\left(\bar{T}_{w}-\bar{T}_{\text {out }}\right)\right]}{Q / A_{T} \Delta T_{m}} \\
\Delta p V / \eta_{f}
\end{array}\right\}
\end{gathered}
$$

Where $\dot{m}$ is mass flow rate of the fluid, $V$ is volume flow rate of the fluid $\left(\mathrm{m}^{3} \mathrm{~s}^{-1}\right), k_{f}$ is the thermal conductivity of the fluid and $\eta_{f}$ is fan efficiency $=0.3[27,29]$. The mean temperature $(\bar{T})$ and mean pressure $(\bar{p})$ for a cross-section are defined as:

$$
\left\{\begin{array}{l}
\bar{T} \\
\bar{p}
\end{array}\right\}=\left\{\begin{array}{c}
\iint_{A} u T d A / \int_{A} u d A \\
\iint_{A} p d A / \iint_{A} d A
\end{array}\right\}
$$

\section{Results and discussion}

\subsection{Model validation and grid independency}

In this study the thermal $(\mathrm{Nu})$ and hydraulic $(f)$ characteristics of the FTHE having RWPs in CFU manner have 
been evaluated at different grid systems for the $R e=700$. The RWPs with different attack angles i.e. $5^{\circ}, 15^{\circ}$ and $25^{\circ}$ are mounted adjacent to the first, third and fifth tube, respectively, $\left(5^{\circ}-15^{\circ}-25^{\circ}\right.$ case $)$. Figure 3 shows the variation of the $\mathrm{Nu}$ and $f$ over the different grid systems having different number of elements $(116955,186540$, $254672,353916,599835$ and 691425). It can be seen in figure 3 that the difference between the predicted results for the grid systems having 599835 and 691425 elements is less than $0.35 \%$. As the difference in the obtained results for thermal and hydraulic characteristics is negligible for the grid system having 599835 and 691425 number of elements, and to reduce the computational efforts the grid system having 599835 number of elements is selected finally for the further predictions. The similar mesh generation strategy has been considered for all the other cases.

The current numerical results are also compared with experimental results given by Joardar and Jacobi [8] over the $R e$ range 500-900. The discrepancy of the current numerical results and the experimental results for the $h$ is $6.59 \%$ (at $R e=500$ ) to $-11.75 \%$ (at $R e=$ 900 ) and that for $\Delta p$ is $5.80 \%$ (at $R e=500$ ) to $-14.70 \%$ (at $R e=900$ ). A little difference between the results exists due to the inevitable thermal resistances and leakage in the experimental process. Moreover, numerical solution approaches the ideal conditions compared to experimental solution. Figure 4 represents a fairly good agreement in the numerical and experimental results which shows the trustworthiness of numerical model for the further analysis. However, the considered performance evaluation criteria i.e. area goodness factor $(j / f)$ and heat transfer rate per unit fan power consumption $\left(Q / P_{f}\right)$ can be derived from the validated numerical results.

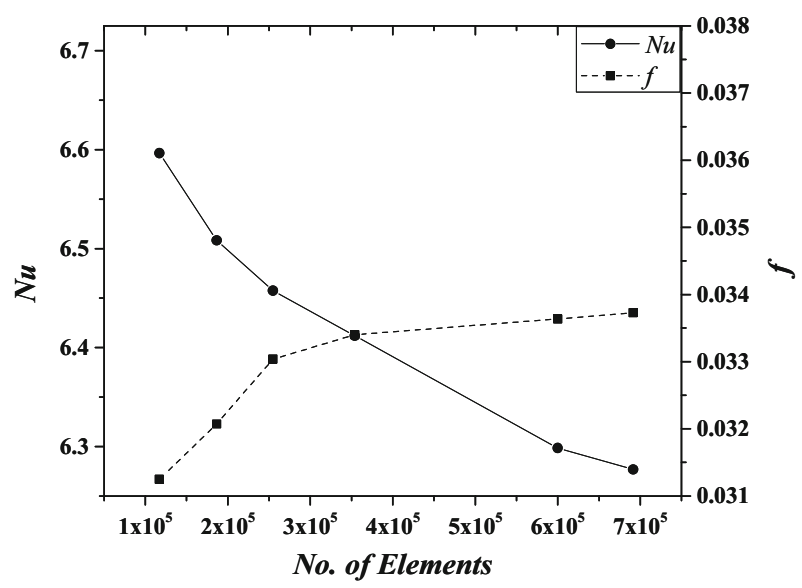

Figure 3. Variation of $N u$ and $f$ with different grid systems.

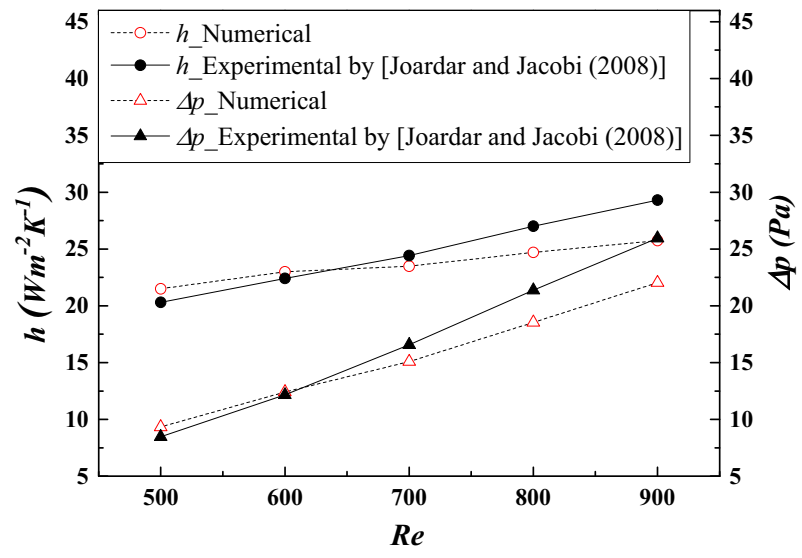

Figure 4. Variation of $h$ and $\Delta p$ with $R e$.

\subsection{Performance evaluation when RWPs with $5^{\circ}$ attack angle are placed at first row}

Generation of the longitudinal vortices is mainly due to the upstream side and the downstream side pressure differences. As the upstream flow moves toward the RWPs, strong swirling flow vortices generate, which interrupt the development of the thermal boundary layer on the fin surface, causes the augmentation in heat transfer rate. Furthermore, placement strategy of RWPs provides a nozzlelike passage adjacent to the tube which increases the heat transfer rate because of separation delay as the fluid flow is accelerated towards the wake zone. In the present numerical investigation RWPs of different attack angles have been mounted adjacent to the tubes alternatively. Vortex generators develop secondary flows in the flow domain by introducing vortices. These vortices generate the strong swirling flow which disrupt the growth of boundary layer and also provide an enhanced mixing of both the fluids (hot and cold) by dragging the cold fluid from the wake zone of the tubes. Moreover, placement strategy and orientation of RWPs can enhance the vortex strength and thus yield additional heat transfer enhancement. In the current study RWPs are arranged in common flow up manner to get the nozzle-like passage adjacent to the tubes.

Figure 5(a) represents the various positions of the cross sections where the velocity contours and velocity stream lines are depicted for the $5^{\circ}-25^{\circ}-15^{\circ}$ case. The same positions of the cross sections have been considered for all other cases $\left(5^{\circ}-15^{\circ}-25^{\circ}, 15^{\circ}-25^{\circ}-5^{\circ}, 15^{\circ}-5^{\circ}-25^{\circ}, 25^{\circ}-5^{\circ}-15^{\circ}\right.$ and $25^{\circ}-15^{\circ}-5^{\circ}$ ) as well. As the flow approaches toward the VGs, wake zone behind the tube is becoming narrower due to the nozzle-like passage created by the VGs. A more converged nozzle-like passage is generated due to high attack angle VGs which intensifies the impingement of fluid on the downstream tube. It can be seen in figures 5(a) and (b) that wake zone behind the tubes is narrower where the high attack angle VGs are mounted, in comparison to the VGs of lower attack angles. When RWP with $25^{\circ}$ attack 


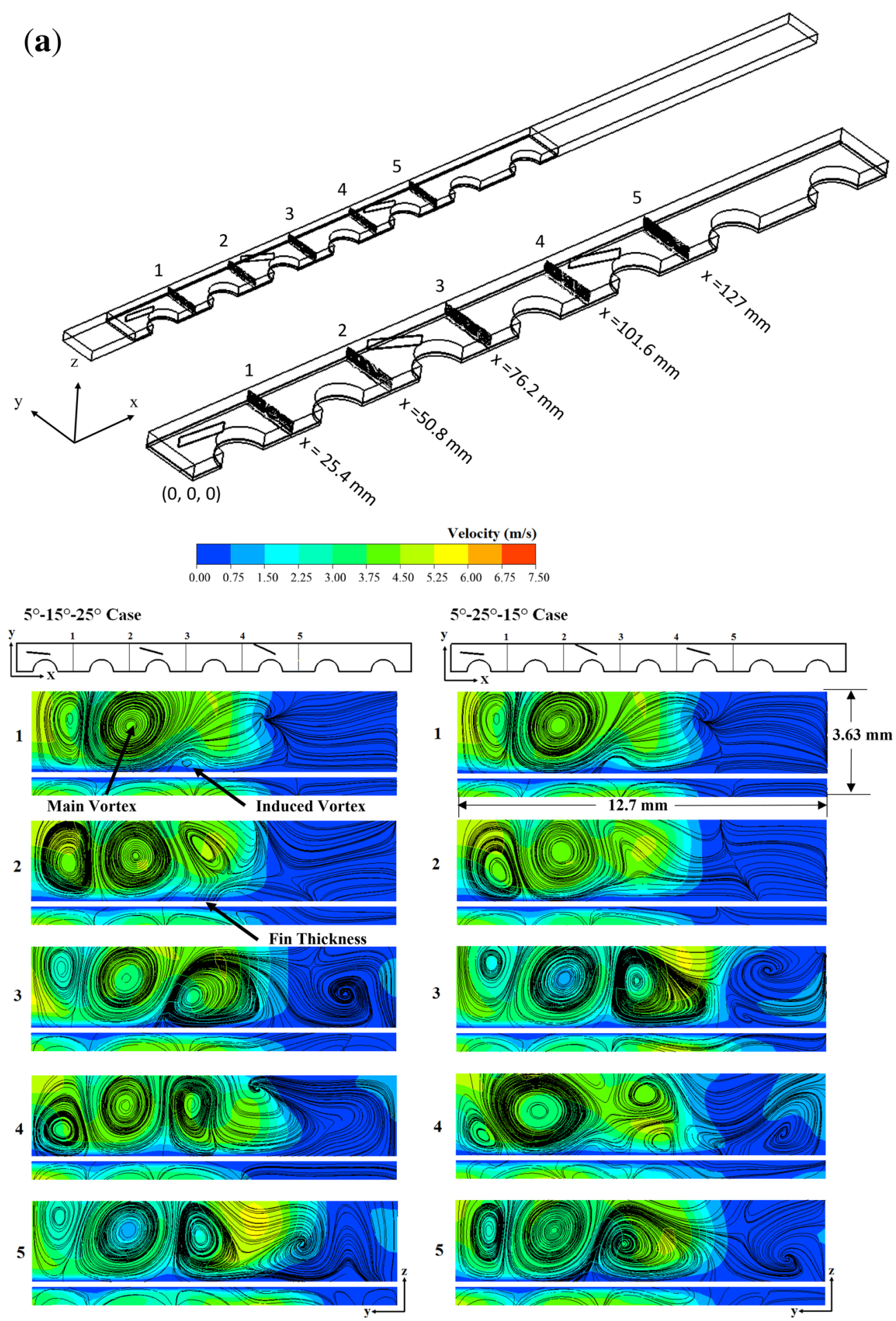

Figure 5. The distribution of velocity and velocity streamlines at (a) different cross-sections and (b) on the middle cross-section of various cases at $R e=900$.

angle is mounted on the third tube, it reduces the wake zone and enhances the mixing of both the fluids which ultimately results in higher local heat transfer coefficients, as compared to the $15^{\circ}$ RWP. The difference between the structures of the vortices can be seen in figure 5(a) at different cross sections. Five different longitudinal positions have been selected for the depiction of vortices formation and interactions to understand the evolution and topology of vortex generation. It can be seen in the streamline depiction of $5^{\circ}-25^{\circ}-15^{\circ}$ case that at position 1 , induced vortex is less significant due to weak interactions between the vortices as the attack angle of winglet is low $\left(5^{\circ}\right)$. It can be appreciated 


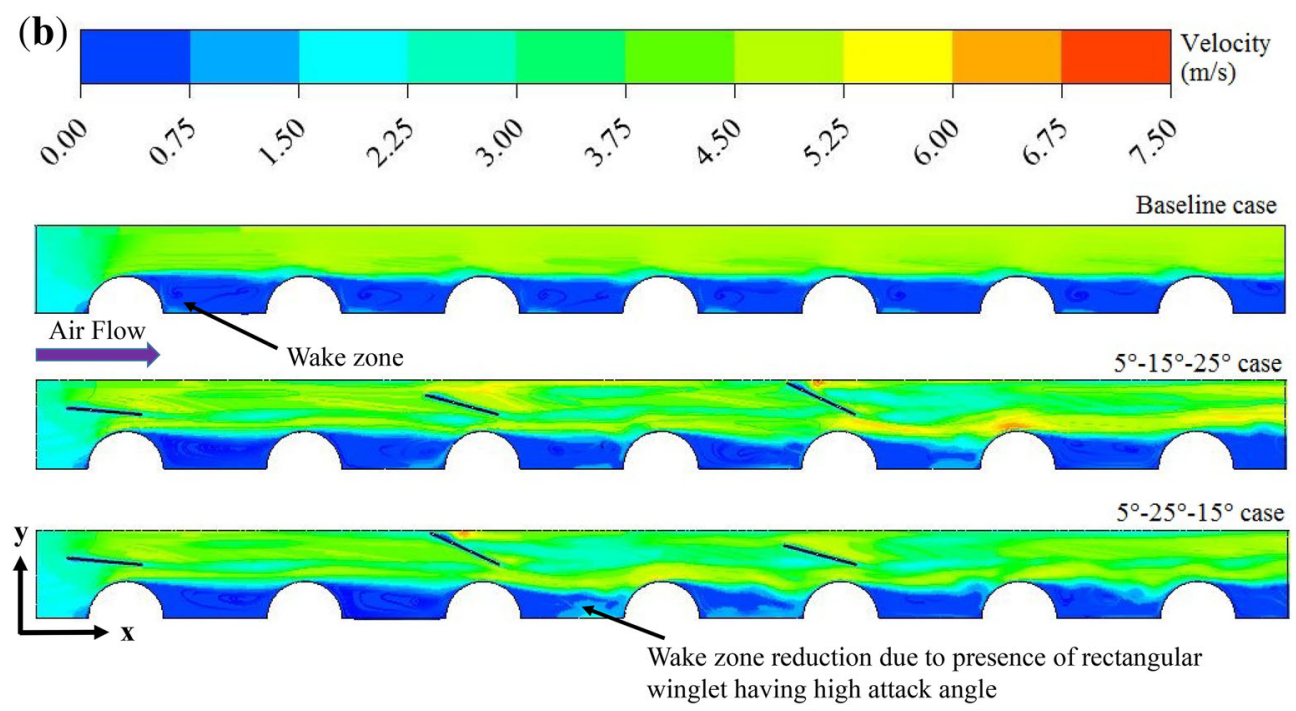

Figure 5. continued

that a strong induced vortex can be generated behind the vortex generators having higher attack angles $\left(15^{\circ}\right.$ or $25^{\circ}$ at position 3 and 5). The formation and interaction of these strong vortices significantly enhanced the mixing of the hot and cold fluid to increase the heat transfer rate. Moreover, the effect of vortex generation also affects the topology of fluid flow behind the adjacent tubes where VGs are not placed (position 2 and 4). It can be seen that at position 2 insignificant formation of induced vortices is noticed, whereas, at position 4, swirls can be appreciated due to the presence of high attack angle VGs before them. These vortices rotate in the opposite directions which enhances the fluid mixing and also drags the wake zone fluid into the main stream flow. The strong interaction of the vortices behind the tubes leads to the narrower wake zone as the vortices try to drag the fluid from wake region to the main flow stream as shown in figure 5(b).

The reduced wake zone and better mixing together enhance the thermal performance of heat exchanger. An improved temperature gradient is achieved behind the tubes by high attack angle VGs as shown in figure 6 . The boundary layer is suppressed by the constricted passage and longitudinal vortices drag the cold fluid from the wake zones to the main flow. All these effects are responsible for enhanced mixing and thus enhanced heat transfer rate. The enhancement in the heat transfer is also associated with the significant increment in pressure drop penalty which increases the friction factor.

Figure 7(a) shows the variation of $N u$ and $f$ with $R e$, when VGs are arranged in such a manner that $5^{\circ}$ attack

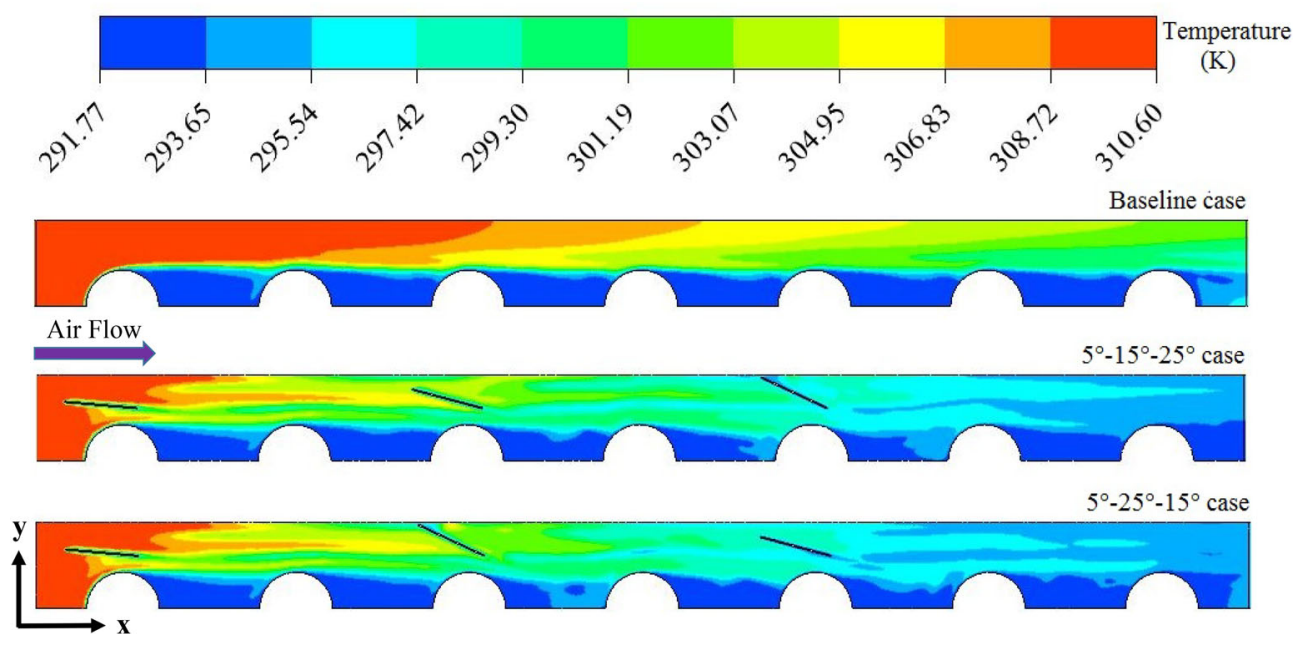

Figure 6. The temperature distribution on the middle cross-section of various cases at $R e=900$. 

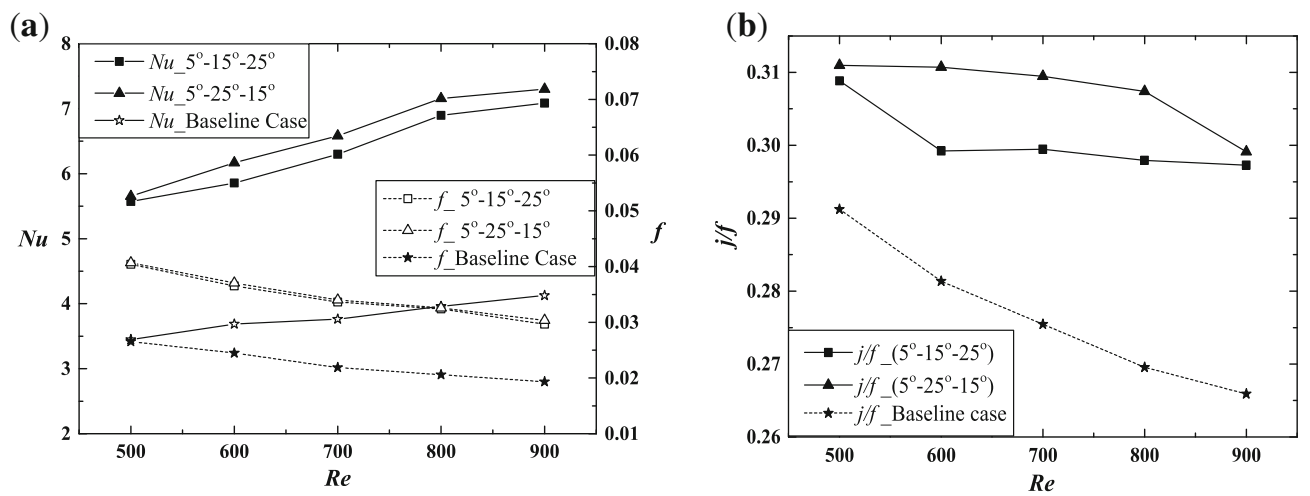

Figure 7. Variation of (a) $N u$ and $f$ with $R e$ and (b) $j / f$ factor with $R e$.

angle RWPs are adjacent to the first tube. It can be seen in the plots, when RWPs of $25^{\circ}$ attack angle are placed adjacent to the third tube, heat transfer rate is higher but at the same time a higher pressure drop penalty also observed, compared to the baseline case. As the difference between the pressure drop penalty of two enhanced cases i.e. $\left(5^{\circ}\right.$ $15^{\circ}-25^{\circ}$ case and $5^{\circ}-25^{\circ}-15^{\circ}$ case) is very less, and increment in $N u$ is significant in $5^{\circ}-25^{\circ}-15^{\circ}$ case which provides the good thermal hydraulic performance of the configuration. Moreover, area goodness factor $(j / f)$ is also calculated for the performance evaluation to get minimal frontal area. Figure 7(b) displays the variation of $j / f$ factor over the considered $R e$ range. It is evident from the plot that these two configurations provide higher values of $j / f$ factor which clearly justify the compactness with better thermal performance. On the basis of area goodness factor $(j / f)$, it can be concluded that $5^{\circ}-25^{\circ}-15^{\circ}$ case provides better thermal hydraulic performance compared to the $5^{\circ}-15^{\circ}-25^{\circ}$ case. And $5^{\circ}-25^{\circ}-15^{\circ}$ case provides an increased $h$ by $68.20 \%$ at $R e=500$ and $81.78 \%$ at $R e=900$ compared to the baseline case i.e., when no VGs are mounted. Moreover, the pressure drop penalty is increased by $76.25 \%$ at $R e=500$ and $72.71 \%$ at $R e=900$ compared to the baseline case.

\subsection{Performance evaluation when RWPs with $15^{\circ}$ attack angle are placed at first row}

In the second arrangement, the RWP having $15^{\circ}$ attack angle is placed at the first row. As the angle of attack increases, streamlines are getting more inclined towards the wake zone behind the tubes and reduce the wakes. It is interesting to put high attack angle RWPs adjacent to the first tube to get more converged like passage. The two possible combinations of RWPs i.e., $15^{\circ}-5^{\circ}-25^{\circ}$ and $15^{\circ}$ $25^{\circ}-5^{\circ}$ are considered for the present numerical analysis. Figure 8(a) depicts the velocity contours and velocity streamlines at various cross sections of $\mathrm{y}-\mathrm{z}$ plane in the flow domain. It is noticed that when the RWP having $5^{\circ}$ attack angle is mounted at the third tube, the longitudinal vortices is seen behind the tubes at the locations $1(\mathrm{X}=$ $25.4 \mathrm{~mm})$ and $2(\mathrm{X}=50.8 \mathrm{~mm})$. Whereas in the second case when the RWP having $5^{\circ}$ attack angle is mounted at the fifth tube the longitudinal vortices is seen at the position $3(X=76.2 \mathrm{~mm})$ and $4(X=101.6 \mathrm{~mm})$. The longitudinal vortices behind the tubes push the fluid from wake zone to the main flow stream which increases the local heat transfer coefficient but with a pressure drop penalty.

Figure 8(b) depicts the local velocity distribution along with the streamlines on the middle plane $(\mathrm{x}-\mathrm{y})$ of crosssection $(\mathrm{y}-\mathrm{z})$ when $R e=900$. It can be appreciated that the wake zone behind the tubes is suppressed by the VGs and the boundary layer is more disrupted where the high attack angle RWPs are mounted as shown in figure 8(a). Hence, a favorable temperature gradient is achieved behind the tube where $25^{\circ}$ attack angle RWP is mounted as shown in figure 9 . It is interesting to note that when $25^{\circ}$ attack angle is placed adjacent to the third tube the effect of longitudinal vortices generation is more visible as shown in figures 8 (b) and figure 9. In this case favourable temperature gradient is obtained behind the third tube which enhances the heat transfer rate as the wake zone is reduced significantly not only behind the third tube but behind the adjacent tube (i.e., tube no. 4) also. On the other hand, when RWP of $25^{\circ}$ attack angle is mounted adjacent to the fifth tube, local $N u$ increases but average $N u$ is found to be less than that of the RWP of $25^{\circ}$ attack angle mounted adjacent to the third tube, as envisioned in figure 10(a). It is also evident that $15^{\circ}-25^{\circ}-5^{\circ}$ case provides higher heat transfer rate compared to both the baseline case and $15^{\circ}$. $5^{\circ}-25^{\circ}$ case but with a significant pressure drop penalty. It is also interesting to note that the rate change of heat transfer is higher than the rate of change in pressure drop at relatively high $R e$ range. It is mainly due to better mixing of the fluid, flow destabilization and obtaining weaker wake zone behind the tubes by incorporating VGs. When $R e$ is higher a strong swirling motion is achieved behind the VGs due to the high strength vortices generation. This high momentum fluid drags the cold fluid from the wake zones to the main flow and thereby heat transfer 
(a)
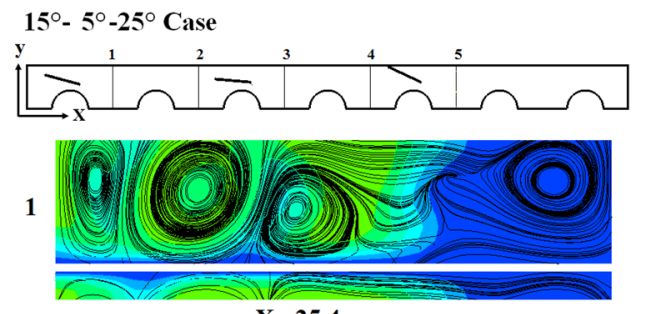

$X=25.4 \mathrm{~mm}$

2

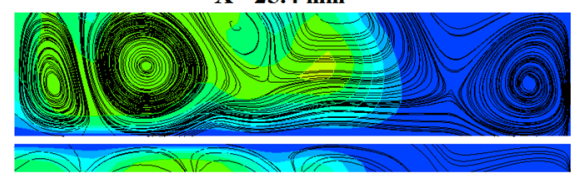

$\mathrm{X}=\mathbf{5 0 . 8} \mathrm{mm}$

3

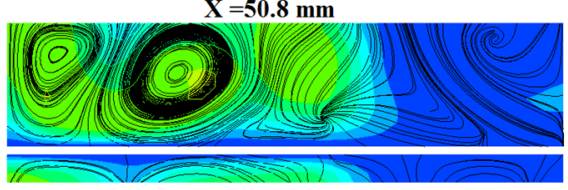

$\mathrm{X}=76.2 \mathrm{~mm}$

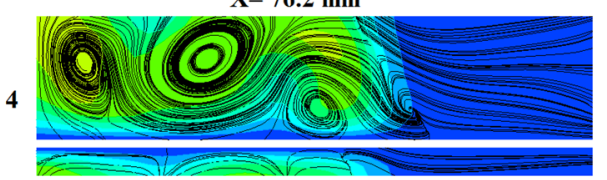

$X=101.6 \mathrm{~mm}$

5

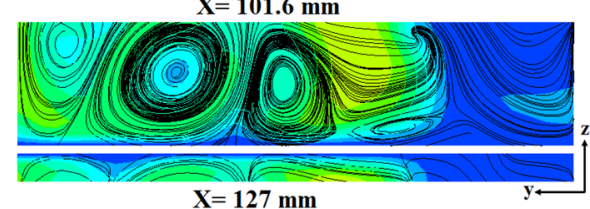

Velocity $(m / s)$

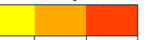

$15^{\circ}-25^{\circ}-5^{\circ}$ Case
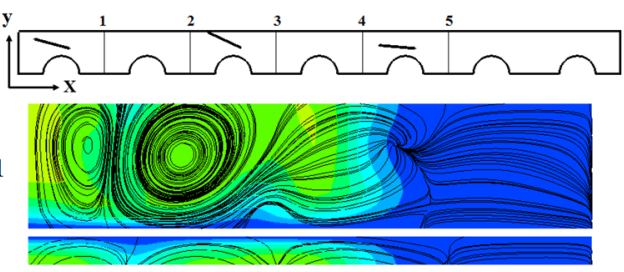

$\mathrm{X}=25.4 \mathrm{~mm}$

2

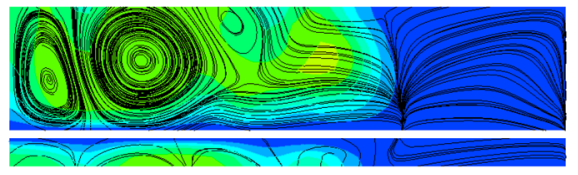

$\mathrm{X}=50.8 \mathrm{~mm}$

3

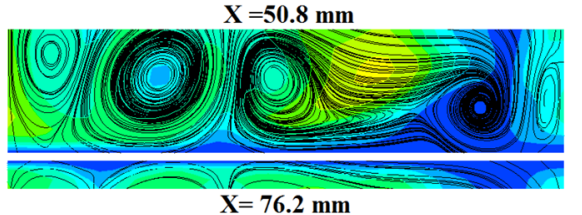

4

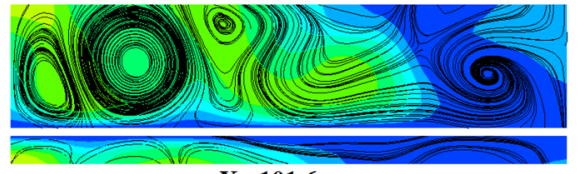

$X=101.6 \mathrm{~mm}$

5

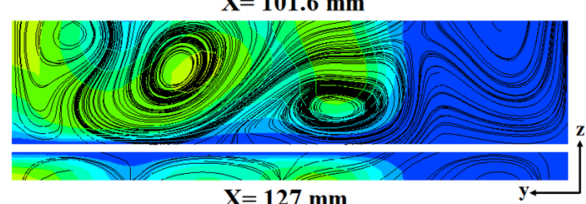

(b)

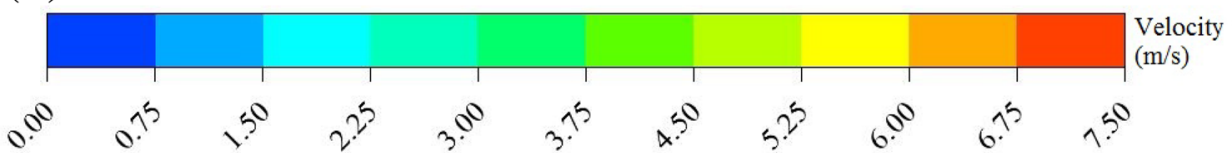

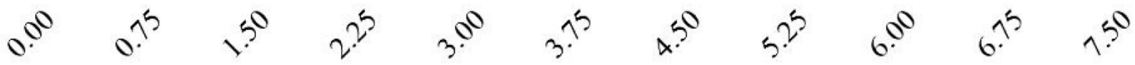
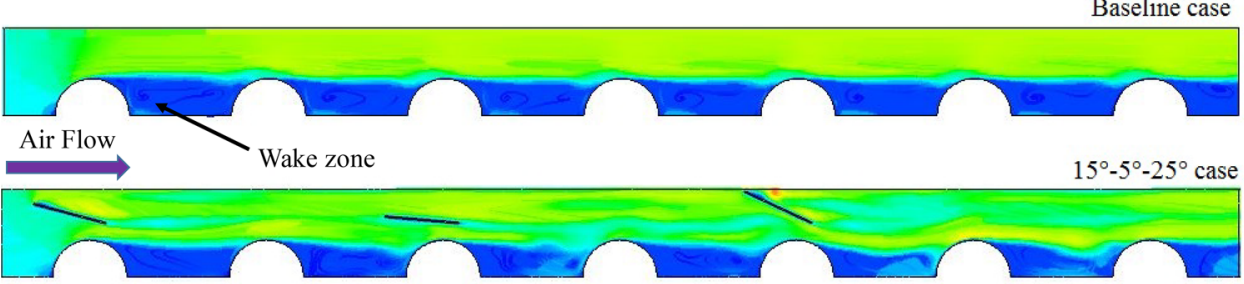

$15^{\circ}-25^{\circ}-5^{\circ}$ case

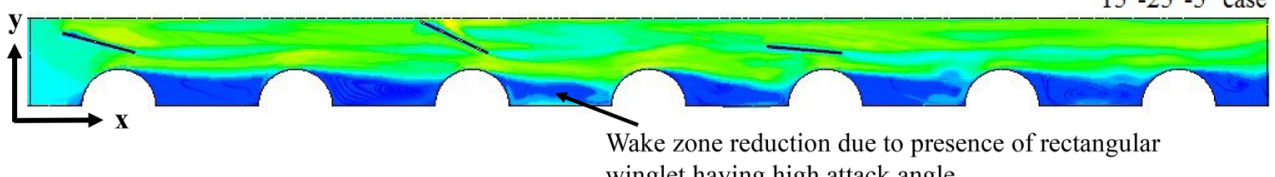

winglet having high attack angle

Figure 8. The distribution of velocity and velocity streamlines at (a) different cross-sections and (b) the middle cross-section of various cases at $R e=900$.

rate is increased. RWP with high attack angle generates the strong vortices at higher $R e$ range that is why a more enhanced heat transfer rate is achieved when $25^{\circ}$ attack angle RWP is mounted adjacent to the third tube compared to the location when it is placed adjacent to the fifth tube.

Figure 10(b) shows the variation of performance evaluation criterion (area goodness factor $(j / f)$ ) over the $R e$ range 


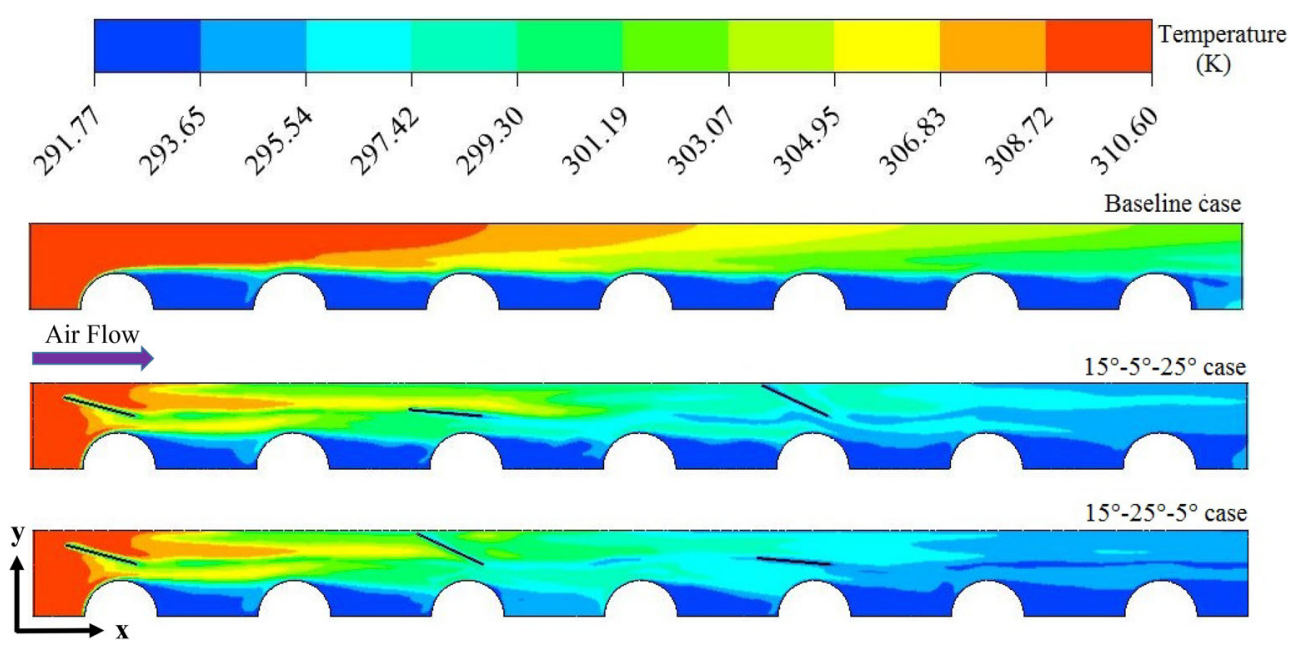

Figure 9. The temperature distribution on the middle cross-section of various cases at $\operatorname{Re}=900$.

(a)

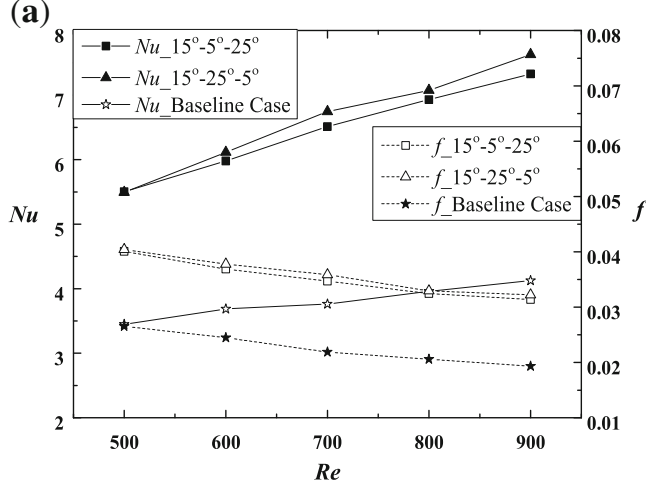

(b)

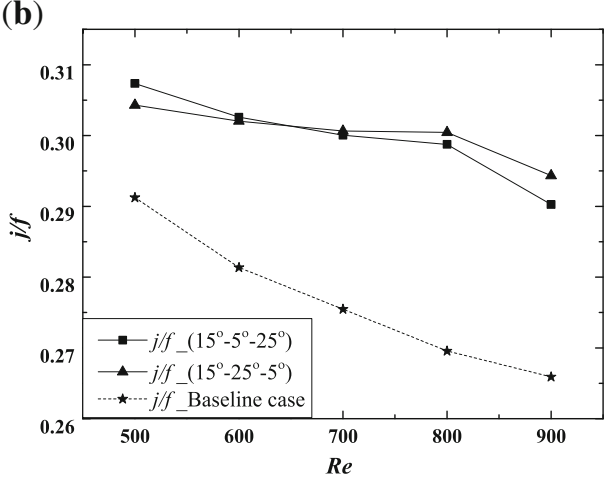

Figure 10. Variation of (a) $N u$ and $f$ with $R e$. (b) Area goodness factor $(j / f)$ with $R e$.

500-900. It is interesting to note that at the lower $R e$ range, $15^{\circ}-5^{\circ}-25^{\circ}$ configuration shows the better thermal hydraulic performance but as the $R e$ increases $15^{\circ}-25^{\circ}-5^{\circ}$ configuration exhibits higher values of performance evaluation criterion $(j / f)$. Therefore, it can be concluded from figures 10 (a) and (b), $15^{\circ}-25^{\circ}-5^{\circ}$ configuration provides minimal frontal area (as the $j / f$ factor is higher) with good thermal hydraulic characteristics and can be chosen as an optimal placement of the VGs compare to both the baseline case and $15^{\circ}-5^{\circ}-25^{\circ}$ configuration. Further, $15^{\circ}-25^{\circ}-5^{\circ}$ configuration provides an increased heat transfer coefficient (h) by $63.48 \%$ at $R e=500$ and $89.93 \%$ at $R e=900$ compared to the baseline case in which no VGs are mounted whereas pressure drop penalty is increased by $75.06 \%$ at $R e$ $=500$ and $83.37 \%$ at $R e=900$ compared to the baseline case. Therefore, it can be noted from the above results that $15^{\circ}-25^{\circ}-5^{\circ}$ case provides higher heat transfer coefficient compared to the $5^{\circ}-25^{\circ}-15^{\circ}$ case at higher $\operatorname{Re}$ but with a significant amount of increased pressure drop penalty.

\subsection{Performance evaluation when RWPs with $25^{\circ}$ attack angle are placed at first row}

In the third arrangement, RWP of $25^{\circ}$ attack angle is mounted adjacent to the first tube, as RWPs with the higher attack angles generate strong longitudinal vortices resulting higher heat transfer rate. Here, two possible combinations i.e. $25^{\circ}-5^{\circ}-15^{\circ}$ case and $25^{\circ}-15^{\circ}-5^{\circ}$ case have been considered. It can be seen in the figure 11(a) that a strong longitudinal vortices zone is obtained behind the first tube (position 1, X=25.4 mm) because of having RWP of higher attack angle $\left(25^{\circ}\right)$. The vortices interaction behind the tubes considerably increases the local heat transfer rate with an appreciable pressure drop penalty.

Higher attack angle converges the flow to the aft of the tube more effectively and thus wake zone behind the tube is reduced as shown in figure 11 . Moreover, it can be seen in figure 12 that RWP of $25^{\circ}$ attack angle generates the favorable temperature gradient behind the first tube but this 
(a)

Velocity $(\mathrm{m} / \mathbf{s})$

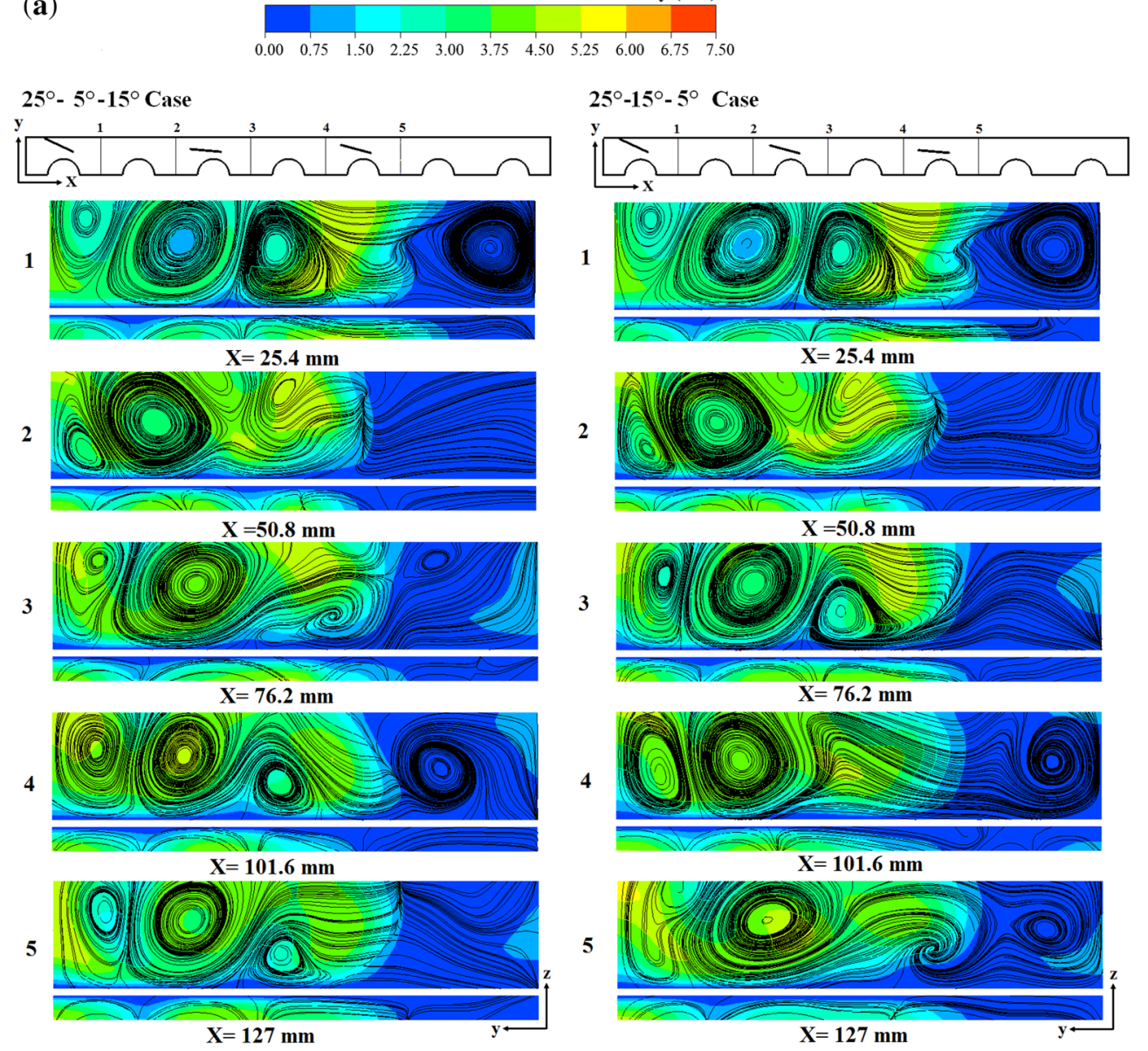

(b)
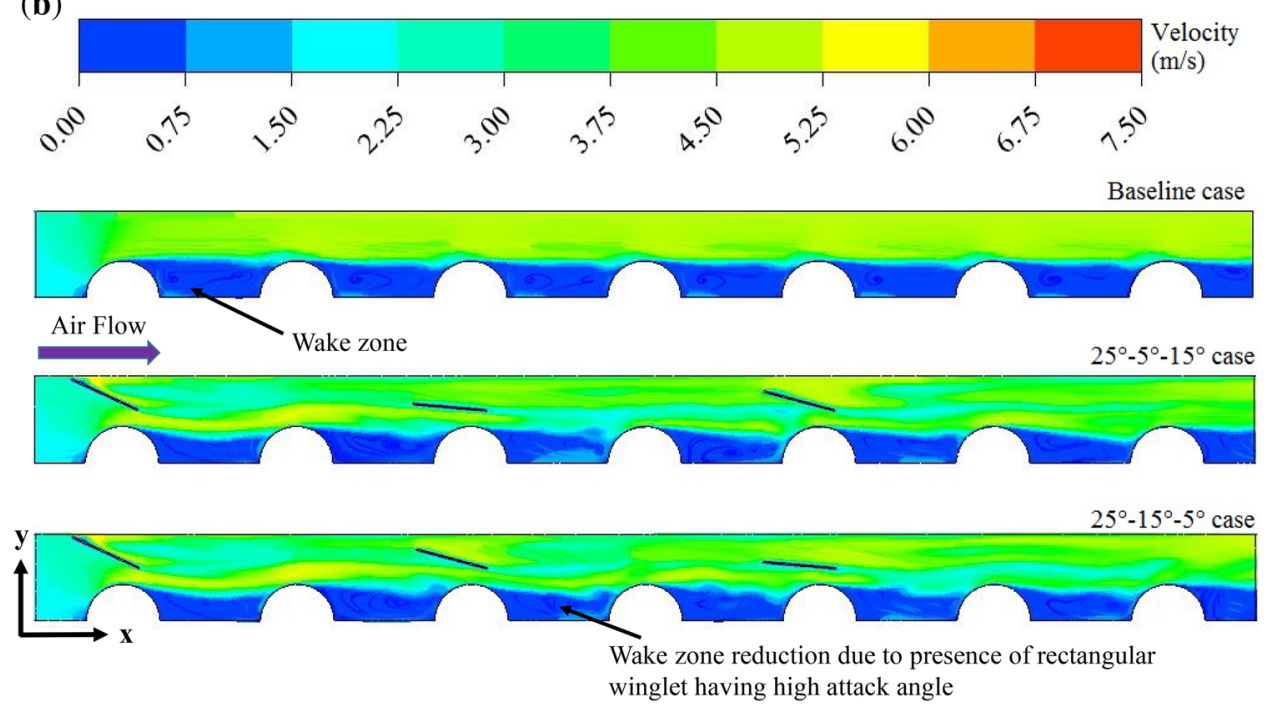

Figure 11. The distribution of velocity and velocity streamlines at (a) different cross-sections and (b) the middle cross-section of various cases at $R e=900$.

effect is less on the other adjacent tubes. RWPs accelerated the fluid into the wake zone which reduces the form drag of tubes. As the angle of attack increases to $25^{\circ}$, the form drag produced by the VGs becomes dominant over the form drag reduction of the tubes which results in higher pressure drop. It is also a worthy point to note that emplacement of the RWP having the higher attack angle $\left(25^{\circ}\right)$ at the first tube causes a strong perturbation in the flow domain which 


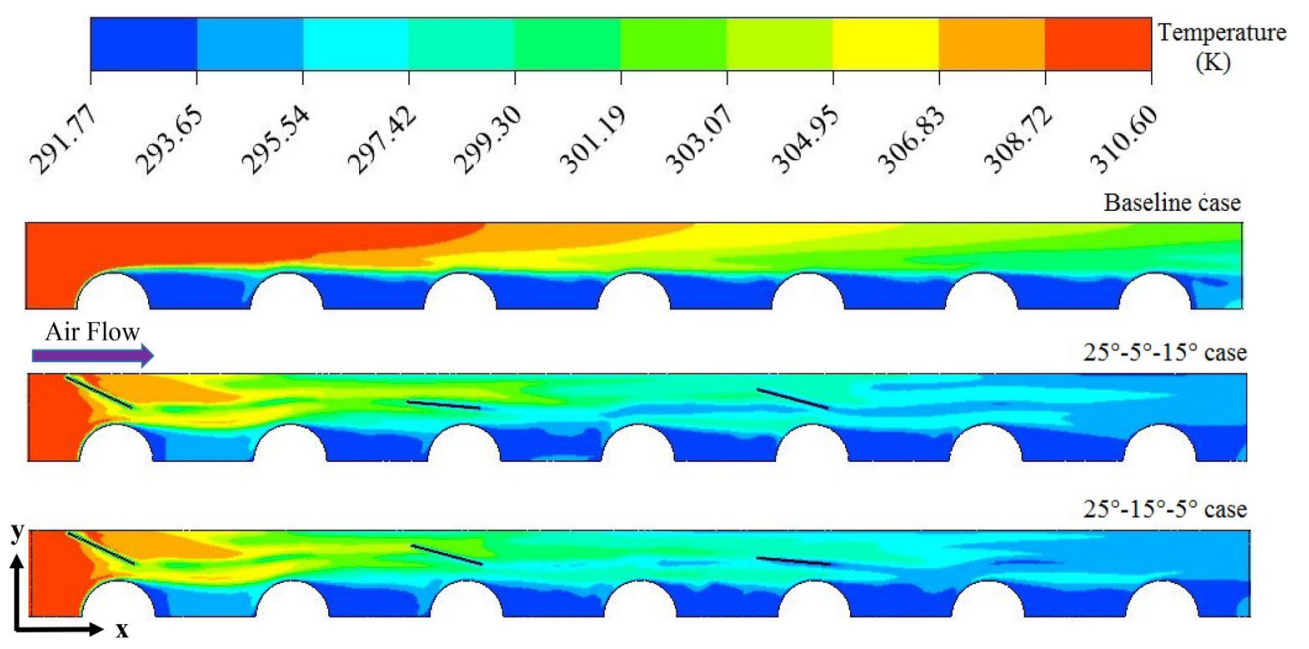

Figure 12. The temperature distribution on the middle cross-section of various cases at $R e=900$.
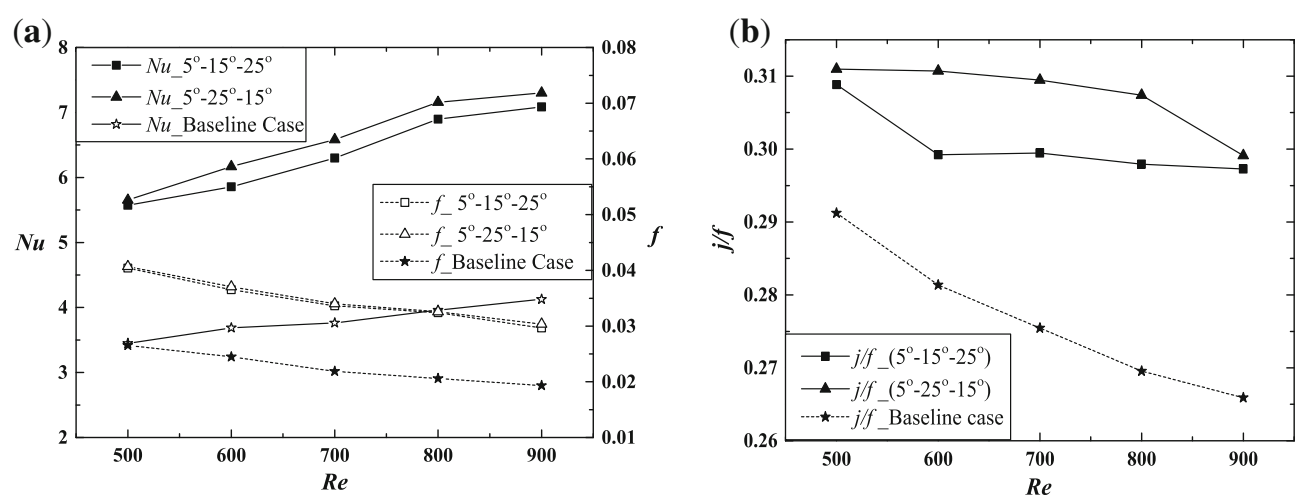

Figure 13. Variation of different parameters (a) $N u$ and $f$ with $R e$. (b) Variation of $j / f$ factor with $R e$.

considerably increases the pressure drop penalty. The increased pressure drop leads to the lower thermal hydraulic performance of the FTHEs. Furthermore, low thermal hydraulic performance of the FTHEs requires the more frontal area for the better heat transfer. Hence, incorporation of the RWPs having high attack angle $\left(25^{\circ}\right)$ at the first row limits the performance and compactness of the FTHEs.

Figure 13 shows the variation of $N u$ and $f$ with $R e$ ranges from 500 to 900 . It can be seen that $25^{\circ}-5^{\circ}-15^{\circ}$ case provides high transfer rate at higher $R e$ values in comparison to that of the $25^{\circ}-15^{\circ}-5^{\circ}$ case. However, the pressure drop is slightly higher in $25^{\circ}-15^{\circ}-5^{\circ}$ case due to consecutive placement of RWPs having high attack angles. Area goodness factor $(j / f)$ is calculated for performance evaluation and represented in figure 13(b). It is evident from the plots that $25^{\circ}-5^{\circ}-15^{\circ}$ case is providing higher values of $j / f$ factor which shows better thermal hydraulic performance with minimal frontal area requirement. The $25^{\circ}-5^{\circ}-15^{\circ}$ case provides an increased $h$ by $56.10 \%$ at $R e=500$ and $78.53 \%$ at $R e=900$ compared to the baseline case whereas pressure drop is increased by $68.81 \%$ at $R e=500$ and $72.6 \%$ at $R e$ $=900$ compared to the baseline case.

The quantitative representation of the area goodness factor for all the considered cases has been summarized in table 1 . It can be concluded from the obtained values of $j / f$ factors that $5^{\circ}-25^{\circ}-15^{\circ}$ case provides the better thermal hydraulic performance in comparison to the considered cases. It is interesting to note that when the winglets having lower attack angle placed in the first row the incoming fluid strikes the fin surface with lesser velocity compared to the high attack angle cases and thus a reduced friction factor is achieved in the low attack angle case. However, in lower attack angle cases, at high $R e$, the thermal performance $\mathrm{Nu}$ ) also deteriorated minutely but due to the reduced pressure drop penalty the enhanced thermal hydraulic performance is obtained. However, an insignificant difference is observed at relatively higher incoming velocities for different combinations of vortex generators having $5^{\circ}$ attack angle winglet at the first row $\left(5^{\circ}-15^{\circ}-25^{\circ}\right.$ and $5^{\circ}-25^{\circ}$ $15^{\circ}$ case) as shown in table 1 . It can be concluded from the above results and discussion that $5^{\circ}-25^{\circ}-15^{\circ}$ case provides 
Table 1. Comparison of Area goodness factor $(j / f)$ values for different attack angle combinations.

\begin{tabular}{lcccrr}
\hline & \multicolumn{5}{c}{ Area goodness factor $(j / f)$} \\
\cline { 2 - 6 } $\operatorname{Re}$ & $5^{\circ}-15^{\circ}-25^{\circ}$ & $5^{\circ}-25^{\circ}-15^{\circ}$ & $15^{\circ}-5^{\circ}-25^{\circ}$ & $15^{\circ}-25^{\circ}-5^{\circ}$ & $25^{\circ}-15^{\circ}-5^{\circ}$ \\
\hline $\mathbf{5 0 0}$ & 0.30884 & $\mathbf{0 . 3 1 0 9 7}$ & 0.30735 & 0.30429 & 0.30132 \\
$\mathbf{6 0 0}$ & 0.29923 & $\mathbf{0 . 3 1 0 6 9}$ & 0.30259 & 0.30203 & 0.29861 \\
$\mathbf{7 0 0}$ & 0.29946 & $\mathbf{0 . 3 0 9 4 6}$ & 0.30004 & 0.30064 & 0.29858 \\
$\mathbf{8 0 0}$ & 0.29793 & $\mathbf{0 . 3 0 7 3 9}$ & 0.29875 & 0.30043 & 0.29500 \\
$\mathbf{9 0 0}$ & 0.29727 & $\mathbf{0 . 2 9 9 1 1}$ & 0.29025 & 0.29434 & 0.29395 \\
\hline
\end{tabular}

Bold values are the highest assessment values and the corresponding configuration is the optimum configuration.

the better thermal hydraulic performance in terms of area goodness factor $(j / f)$ compared to other considered cases. Furthermore, heat transfer rate per unit fan power consumption $\left(Q / P_{f}\right)$ is also introduced as another performance evaluation criterion (PEC). It can be seen in the depiction of the heat transfer rate per unit fan power consumption $\left(Q / P_{f}\right)$ that all the cases of having RWPs are showing lower performance compared to the baseline case as shown in figure 14. This is mainly due to the high pressure loss which is contributed by the RWPs.

However, the cases when RWPs having $25^{\circ}$ attack angle are placed in the first rows provide the higher $Q / P_{f}$ values compared to the other cases due to the higher heat transfer rate. It is also to be mentioned that the $Q / P_{f}$ values for all the considered cases are inferior with respect to the baseline case due to high fan power consumption.

However, when the compactness ( $j / f$ factor) and the thermal performance $(\mathrm{Nu})$ are the main concerned, the FTHEs having RWPs promise a significant increment in the thermo-hydraulic performance of FTHEs.

\subsection{MOORA analysis}

However, the present numerical investigation is divided into three subsections on the basis of fixing attack angle of RWPs adjacent to the first tube. The optimal configuration can be obtained by the $j / f$ factor values over the considered $R e$ range. It can be concluded from the above discussions that the $5^{\circ}-25^{\circ}-15^{\circ}$ case can be chosen as an optimal configuration on the basis of high thermal hydraulic performance with minimal frontal area requirement. Furthermore, MOORA method is incorporated to get and check the performance order of the various configurations. In MOORA, performance ranking $\left(y_{i}\right)$ can be obtained as:

$$
y_{i}=\sum_{j=1}^{g} x_{i j}^{*}-\sum_{j=g+1}^{n} x_{i j}^{*}
$$

The normalized performance measure $\left(x_{i j}^{*}\right)$ is defined as:

$$
x_{i j}^{*}=x_{i j} / \sum_{i=1}^{m} x_{i j}
$$

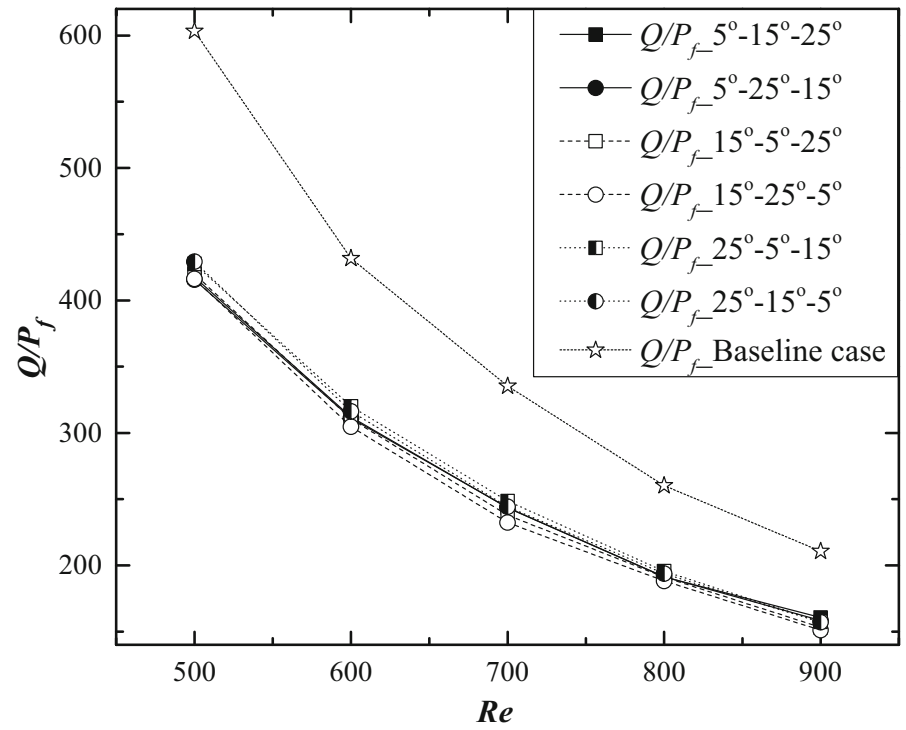

Figure 14. Variation of heat transfer rate per unit fan power consumption over the $R e$ range (500-900). 
Table 2. Assessment values obtained by MOORA method for different attack angle combinations.

\begin{tabular}{|c|c|c|c|c|c|c|c|}
\hline \multirow[b]{2}{*}{$R e$} & \multicolumn{6}{|c|}{ Assessment Values $\left(\mathrm{y}_{\mathrm{i}}\right)$} & \multirow[b]{2}{*}{ Baseline case } \\
\hline & $5^{\circ}-15^{\circ}-25^{\circ}$ & $5^{\circ}-25^{\circ}-15^{\circ}$ & $15^{\circ}-5^{\circ}-25^{\circ}$ & $15^{\circ}-25^{\circ}-5^{\circ}$ & $25^{\circ}-5^{\circ}-15^{\circ}$ & $25^{\circ}-15^{\circ}-5^{\circ}$ & \\
\hline 500 & 0.0486 & 0.0493 & 0.0482 & 0.0472 & 0.0463 & 0.0467 & 0.0437 \\
\hline 600 & 0.0473 & 0.0511 & 0.0484 & 0.0483 & 0.0471 & 0.0452 & 0.0425 \\
\hline 700 & 0.0479 & 0.0512 & 0.0481 & 0.0483 & 0.0476 & 0.0456 & 0.0413 \\
\hline 800 & 0.0481 & 0.0512 & 0.0484 & 0.0490 & 0.0472 & 0.0458 & 0.0403 \\
\hline 900 & 0.0493 & 0.0500 & 0.0470 & 0.0484 & 0.0483 & 0.0465 & 0.0405 \\
\hline
\end{tabular}

Bold values are the highest assessment values and the corresponding configuration is the optimum configuration.

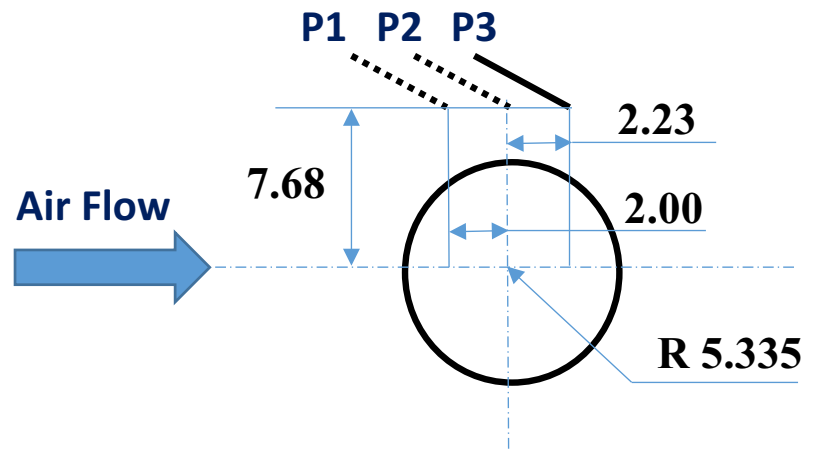

Figure 15. Geometric description of the various positions of RWPs (all dimensions are in $\mathrm{mm}$ ).

Where $x_{i j}$ is the performance measure of $i^{t h}$ alternative on $j^{\text {th }}$ criterion, $m$ is the number of alternatives and $n$ is the number of criterion, $g$ is the number of criteria to be maximized, $(n-g)$ is the number of criteria to be minimized. The highest value of the $y_{i}$ shows the best alternative.

The sole objective of the present MOORA analysis is to obtain a configuration of FTHE which can provide high thermal performance $(\mathrm{Nu})$ and high compactness ( $\mathrm{j} / \mathrm{f}$ factor) with reduced friction factor $(f)$. That is why, in the present analysis Nusselt number $(\mathrm{Nu})$ and area goodness factor $(\mathrm{j} / \mathrm{f})$ have been taken as beneficial attributes whereas friction factor $(f)$ has been taken as a non-beneficial attribute having equal significance to each of the attributes. Table 2 shows the assessment values obtained by the MOORA method. It can be seen in the table that assessment values obtained by the $5^{\circ}-25^{\circ}-15^{\circ}$ case is higher in comparison to the other considered cases. Hence, MOORA method also confirms that the $5^{\circ}-25^{\circ}-15^{\circ}$ case can be chosen as an optimal configuration on the basis of considered performance evaluation criteria.

\subsection{Effect of longitudinal position of RWPs on the performance of FTHE}

It can be concluded from the above results and discussion that the configuration of FTHE having RWPs as mentioned in $5^{\circ}-25^{\circ}-15^{\circ}$ case provides the higher performance compared to the other considered cases. The trailing edge of the rectangular winglet is fixed at a distance of $2.23 \mathrm{~mm}$ from the centre of the tube in downstream region (P3) as shown in figure 15. Furthermore, it is interesting to analyse the effect of trailing edge position on the performance of FTHE. In the present numerical analysis, three different positions $\mathrm{P} 1, \mathrm{P} 2$ and $\mathrm{P} 3$ are considered for the analysis as shown in figure 15 .

It can be seen in the velocity distributions (figure 16a) that position 2 of winglet provides the more effective nozzle-like effect compared to the position 1 . Thus, it impinges the fluid with high velocity to the second tube which significantly enhances the heat transfer coefficient which results high thermal performance. A better temperature gradient is achieved behind the first and second tubes in case when winglet is placed at position 2 as shown in figure 16(b). It is also important to note that in position 2 case, the high accelerated fluid strikes the fin surface with high velocity and contributes in the increment of friction factor which lowers the hydraulic performance of the heat exchanger. However, Position 1 provides the lesser thermal performance compared to the Position 2 but due to the less friction factor it significantly provides the better thermal hydraulic performance ( $j / f$ factor and $Q / P_{f}$ factor) compared to the other considered positions of winglet.

The thermal characteristics in terms of Nusselt number $\mathrm{Nu}$ and hydraulic characteristics in terms of friction factor $(f)$ have been depicted in figure 15 for the $5^{\circ}-25^{\circ}-15^{\circ}$ case at $R e=700$. It can be seen in figure 17 that at position 2 which is aligned to the centre of tube provides the higher values of Nusselt number with a significant increment in the pressure loss. Whereas, at the position 1 when the trailing edge of the RWP is kept in the upstream region a marginal increment in the Nusselt number $(\mathrm{Nu})$ is achieved with a significant reduction in the friction factor $(f)$. The area goodness factor $(j / f)$ at various positions has been depicted in figure 18. It can be seen that position 1 provides a higher area goodness factor (3.93\% higher than position 3 ) which clearly shows a good compactness in comparison to the other considered positions. This is mainly due to the high thermal performance with a reduced friction factor compared to the previous position (position 3). Another performance evaluation criterion i.e. heat transfer rate per unit fan power consumption $\left(Q / P_{f}\right)$ is also presented in 
(a)
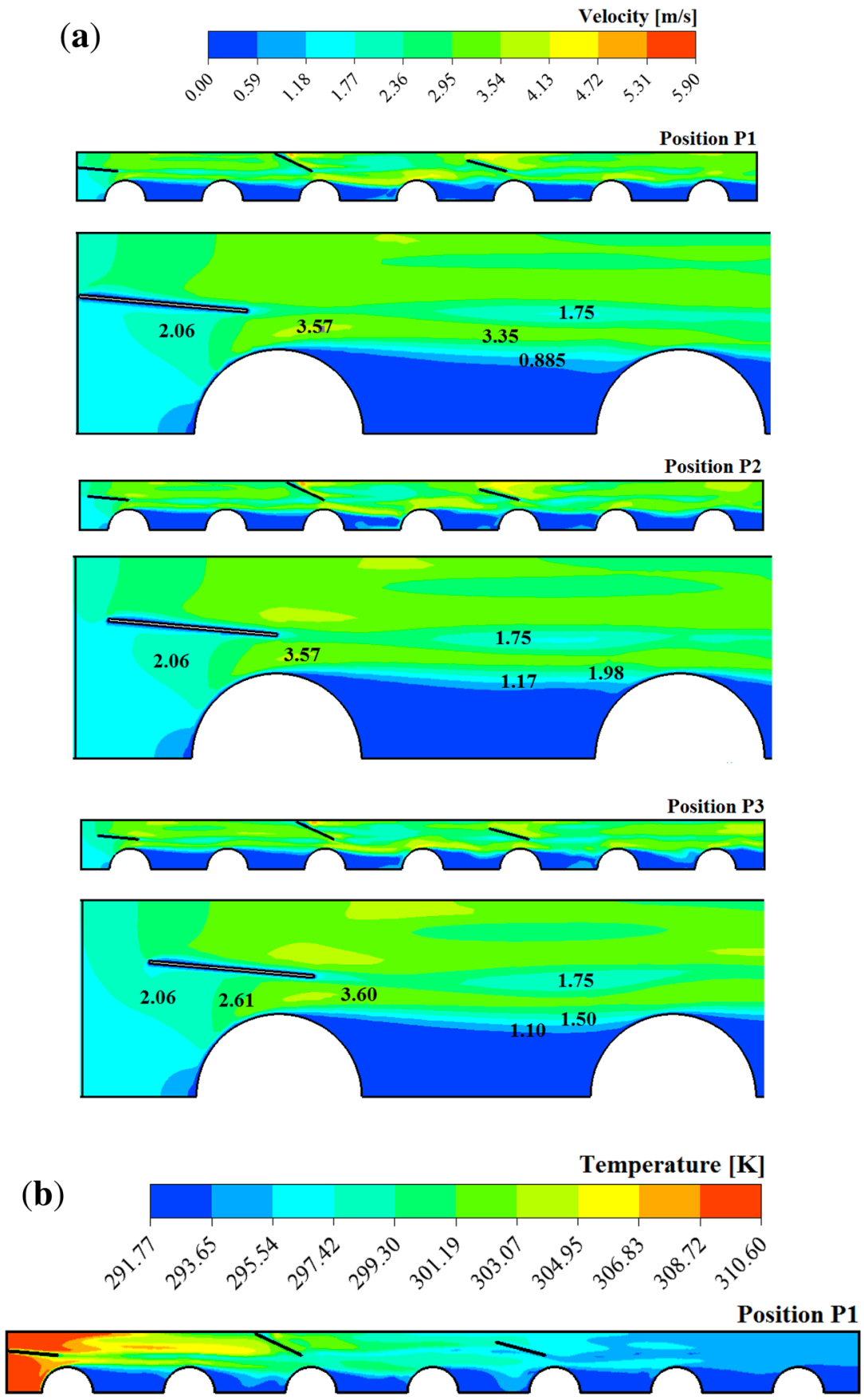

Position P2

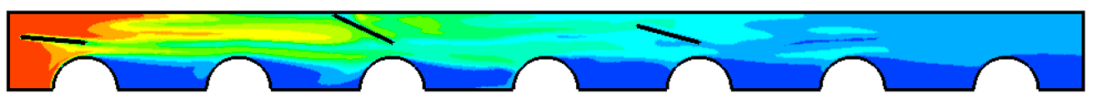

Position P3

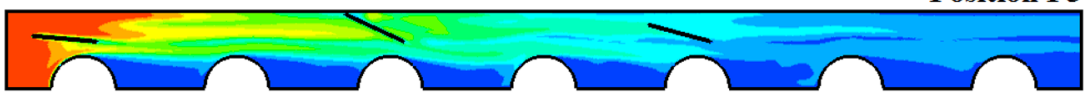

Figure 16. Distribution of (a) velocity and (b) temperature at mid plane of the computational domain for $5^{\circ}-25^{\circ}-15^{\circ}$ case $($ at $R e=700$ ).

figure 19. It can be noticed that heat transfer rate per unit fan power consumption is also higher at position 1 compared to the previously taken position (position 3). The position 2 shows the lower performances in terms of area goodness factor and heat transfer rate per unit fan power consumption due to the high pressure loss compared to 


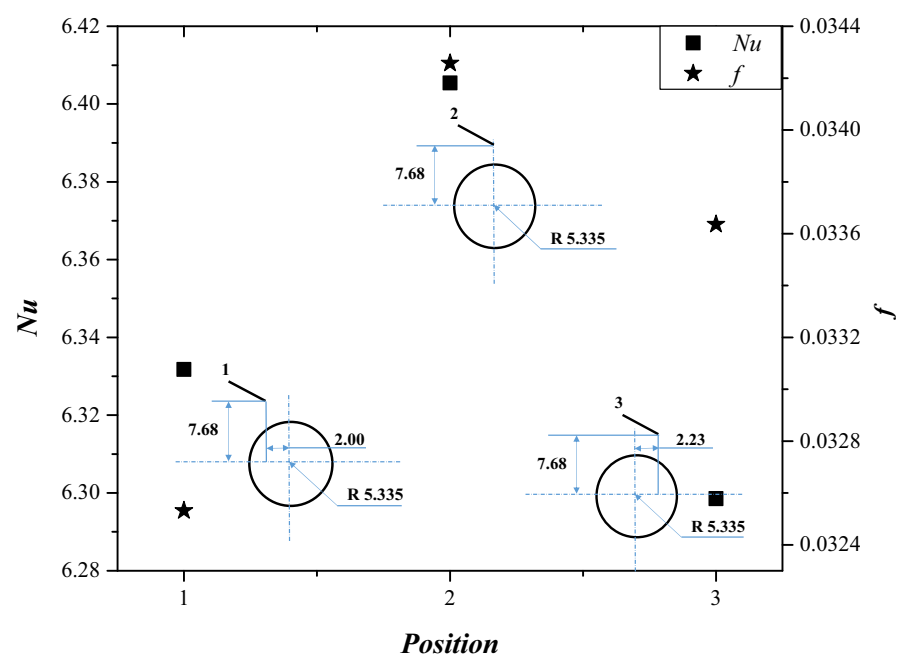

Figure 17. Variation of $N u$ and $f$ with respect to various positions at $R e=700$ (all dimensions are in $\mathrm{mm}$ ).

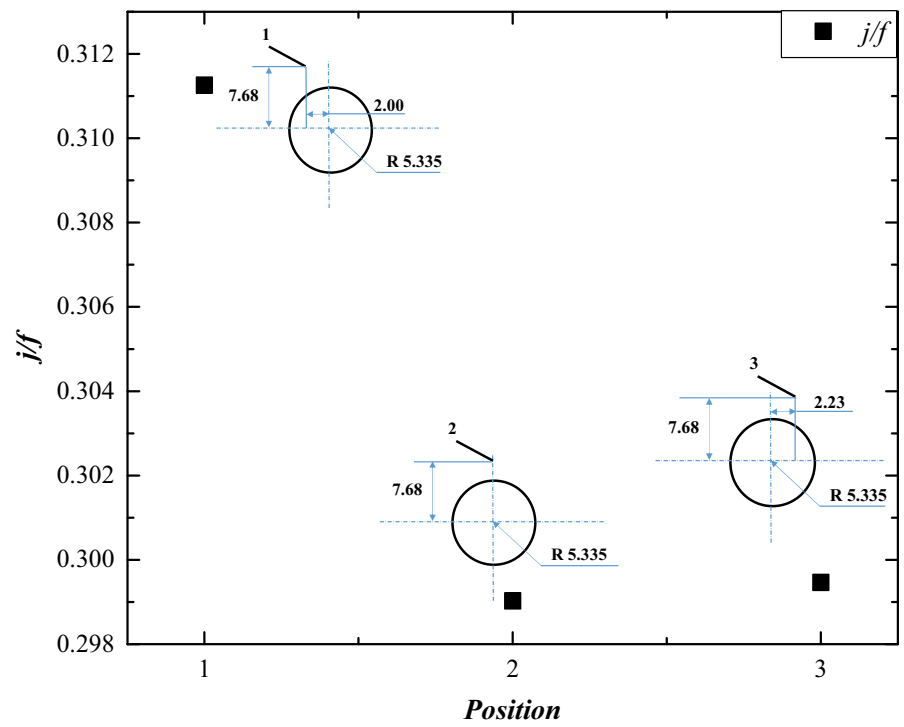

Figure 18. Variation of $j / f$ factor with respect to various positions at $R e=700$ (all dimensions are in $\mathrm{mm}$ ).

other considered positions. Finally, it can be concluded that position 1 is the best location to get high thermal hydraulic performance.

\section{Conclusion}

In the present numerical analysis totally six combinations of RWPs with different attack angles have been analyzed. The performance evaluation has been done by $j / f$ factor. MOORA method is also incorporated for getting performance ranking by taking $N u$ and $j / f$ factor as beneficial attributes and $f$ as a non-beneficial attribute, with each attribute having equal importance. The major findings of this study are as follows:

- Placement of VGs with different attack angles affects the thermal hydraulic behaviour of the fluid. It is found that combinations of VGs having different attack angles provide enhanced $j / f$ factor corresponding to the baseline case which ensures the requirement of minimum frontal area. Moreover, all the combinations provide higher heat transfer rate compared to base line case but with a significant pressure drop penalty.

- Results indicate that placement of VGs with the lowest attack angle adjacent to the first row tubes provides good thermal hydraulic performance compared to other 


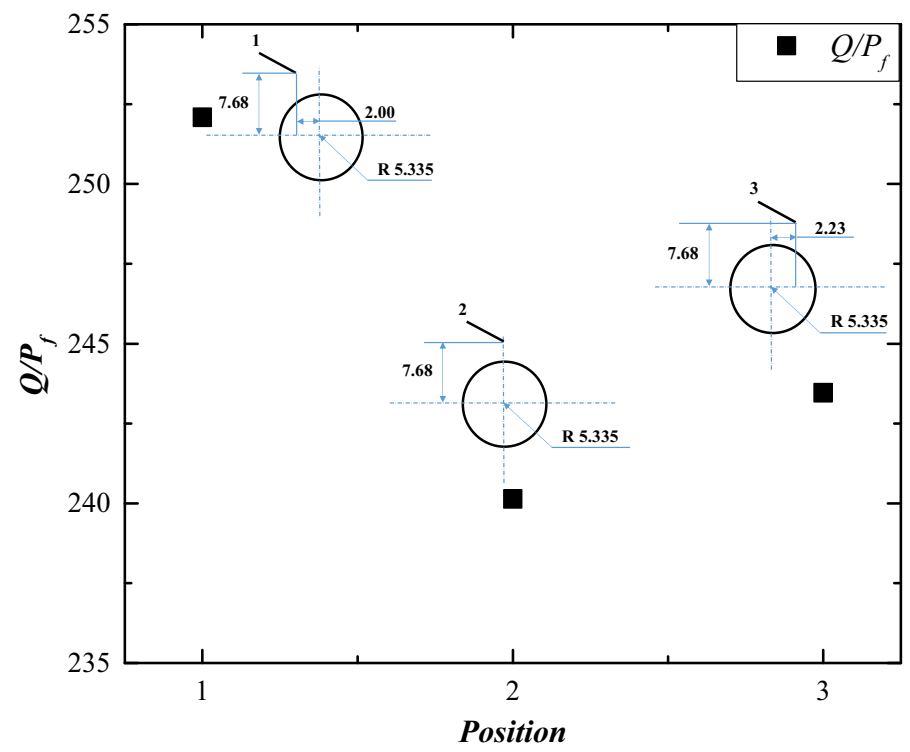

Figure 19. Variation of $Q / P_{f}$ factor with respect to various positions at $R e=700$ (all dimensions are in $\mathrm{mm}$ ).

cases. It is also found that the placement of RWPs with $25^{\circ}$ attack angle adjacent to the third tube is more favorable for the better performance in comparison to the placing it at the first or the third tube.

- Higher area goodness factor $(j / f)$ values show that $5^{\circ}$ $25^{\circ}-15^{\circ}$ case is an optimal configuration of FTHE, where $j / f$ factor is increased by $6.77 \%$ at $R e=500$ and $12.49 \%$ at $R e=900$ compared to the baseline case.

- Furthermore, performance ranking obtained by the MOORA method also ensures $5^{\circ}-25^{\circ}-15^{\circ}$ configuration is the optimum one, as it provides the highest assessment values. The configuration $5^{\circ}-25^{\circ}-15^{\circ}$ provides an increased $h$ by $68.20 \%$ at $R e=500$ and $81.78 \%$ at $R e=900$ in comparison to the baseline case in which no VGs are mounted with a pressure drop penalty increased by $76.25 \%$ at $R e=500$ and $72.71 \%$ at $R e=900$ compared to the baseline case.

- An enhanced thermal hydraulic performance of FTHE having RWPs in $5^{\circ}-25^{\circ}-15^{\circ}$ configuration is found when the trailing edge of the rectangular winglet is placed at the position 2 (in the upstream region, $2 \mathrm{~mm}$ from the center of the tube) compared to the other considered positions.

The present analysis of performance evaluation and optimization can be utilised in design and selection of the fin and tube heat exchangers having RWPs. The current optimized results are based on the considered beneficial and non-beneficial attributes. However, further optimization can be done by considering other parameters as beneficial (heat transfer coefficient, heat transfer rate per unit fan power consumption, Colburn factor, etc.) and non-beneficial attributes (pressure drop, fan power, etc.).

\section{Nomenclature}

$A_{T} \quad$ total heat transfer surface area $\left(\mathrm{m}^{2}\right)$

$A_{\text {min }}$ minimum flow area $\left(\mathrm{m}^{2}\right)$

$c_{p} \quad$ specific heat $\left(\mathrm{J} \mathrm{kg}^{-1} \mathrm{~K}^{-1}\right)$

$D \quad$ outer tube diameter $(\mathrm{m})$

$D_{h} \quad$ hydraulic diameter, $\mathrm{D}_{\mathrm{h}}=4 \mathrm{~A}_{\min } \mathrm{L} / \mathrm{A}_{\mathrm{T}}$

$f \quad$ friction factor

$F_{p} \quad$ fin pitch (m)

$F_{t} \quad$ fin thickness (m)

$h \quad$ air-side heat transfer coefficient $\left(\mathrm{W} \mathrm{m}{ }^{-2} \mathrm{~K}^{-1}\right)$

$H \quad$ channel height (m)

$H_{w} \quad$ winglet height (m)

$j \quad$ Colburn j-factor

$L \quad$ flow length (m)

$m$ mass flow rate $(\mathrm{kg} / \mathrm{s})$

$\mathrm{Nu}$ average Nusselt number

$p \quad$ pressure $(\mathrm{Pa})$

$P_{f} \quad$ fan power (W)

$P r \quad$ Prandtl number

$P_{l} \quad$ longitudinal tube pitch (m)

$P_{s} \quad$ span wise tube pitch (m)

$Q \quad$ heat transfer capacity (W)

$\mathrm{Re} \quad$ air side Reynolds number

St Stanton number

$T$ temperature (K)

$T_{\text {out }}$ outlet temperature (K)

$T_{w} \quad$ wall temperature (K)

$\bar{T} \quad$ bulk average temperature $(\mathrm{K})$

$T_{i n} \quad$ inlet temperature (K)

$\Delta T_{m}$ mean value of temperature

$\bar{p} \quad$ bulk average pressure $(\mathrm{Pa})$

$\Delta p \quad$ air side pressure drop $(\mathrm{Pa})$

$U$ free stream velocity $\left(\mathrm{ms}^{-1}\right)$ 
$u \quad$ velocity in $\mathrm{x}$-direction $\left(\mathrm{ms}^{-1}\right)$

$v \quad$ velocity in y-direction $\left(\mathrm{ms}^{-1}\right)$

$V_{m} \quad$ mean velocity at $\mathrm{A}_{\min }\left(\mathrm{m} \mathrm{s}^{-1}\right)$

$w \quad$ velocity in $\mathrm{z}$-direction $\left(\mathrm{m} \mathrm{s}^{-1}\right)$

\section{Greek symbols}

$\mu \quad$ dynamic viscosity ( $\mathrm{Pa} . \mathrm{S}$ )

$\rho$ density $\left(\mathrm{kg} \mathrm{m}^{-3}\right)$

$\lambda$ thermal conductivity $\left(\mathrm{W} \mathrm{m}{ }^{-1} \mathrm{~K}^{-1}\right.$ )

$\eta_{f}$ fan efficiency

$\begin{array}{ll}\text { Abbreviations } \\ \text { FTHE } & \begin{array}{l}\text { finned tube heat exchanger } \\ \text { multi-objective optimization on the basis of } \\ \text { MOORA }\end{array} \\ & \begin{array}{l}\text { ratio analysis } \\ \text { rectangular winglet pair }\end{array} \\ \text { RWP } & \text { longitudinal vortex generators } \\ \text { LVG } & \text { common flow down } \\ \text { CFD } & \text { common flow up } \\ \text { CFU } & \end{array}$

\section{References}

[1] Jacobi A M and Shah R K 1995 Heat transfer surface enhancement through the use of longitudinal vortices: a review of recent progress. Experimental Thermal and Fluid Science 11: 295-309

[2] Bendaoud A L, Ouzzane M, Aidoun Z and Galanis N 2011 A novel approach to study the performance of finned-tube heat exchangers under frosting conditions. Journal of Applied Fluid Mechanics 4: 9-20

[3] Yoo S Y, Park D S and Chung M H 2002 Heat transfer enhancement for fin-tube heat exchanger using vortex generators. KSME International Journal 16: 109-115

[4] Chu P, He Y L, Lei Y G, Tian L T and Li R 2009 Threedimensional numerical study on fin-and-oval-tube heat exchanger with longitudinal vortex generators. Applied Thermal Engineering 29: 859-876

[5] Huisseune H, Joen C T, Jaeger P, De Ameel B, Schampheleire S D and Paepe M D 2013 Influence of the louver and delta winglet geometry on the thermal hydraulic performance of a compound heat exchanger. International Journal of Heat and Mass Transfer 57: 58-72

[6] Tiwari S, Maurya D and Eswaran V 2003 Heat transfer enhancement in cross-flow heat exchangers using oval tubes and multiple delta winglets. International Journal of Heat and Mass Transfer 46: 2841-2856

[7] Leu J S, Wu Y H and Jang J Y 2004 Heat transfer and fluid flow analysis in plate-fin and tube heat exchangers with a pair of block shape vortex generators. International Journal of Heat and Mass Transfer 47: 4327-4338

[8] Joardar A and Jacobi A M 2008 Heat transfer enhancement by winglet-type vortex generator arrays in compact plain-finand-tube heat exchangers. International Journal of Refrigeration 31: 87-97

[9] Kumar A, Joshi J B, Nayak A K and Vijayan P K 2015 A review on the thermal hydraulic characteristics of the air-cooled heat exchangers in forced convection. Sādhanā 3(40): 673-755
[10] Arshad H, Khushnood S, Nizam L A, Ahsan M A and Bhatti O G 2018 Effect of fin geometry on flow-induced vibration response of a finned tube in a tube bundle. Journal of Applied Fluid Mechanics 11(40): 1143-1152

[11] Wu J M and Tao W Q 2008 Numerical study on laminar convection heat transfer in a rectangular channel with longitudinal vortex generator. Part A: Verification of field synergy principle. International Journal of Heat and Mass Transfer 51: 1179-1191

[12] Lin C N, Liu Y W and Leu J S 2008 Heat transfer and fluid flow analysis for plate-fin and oval tube heat exchangers with vortex generators. Heat Transfer Engineering 29(7): 588-596

[13] He Y L, Han H, Tao W Q and Zhang Y W 2012 Numerical study of heat-transfer enhancement by punched winglet-type vortex generator arrays in fin-and-tube heat exchangers. International Journal of Heat and Mass Transfer 55: 5449-5458

[14] He Y L, Chu P, Tao W, Zhang Y W and Xie T 2013 Analysis of heat transfer and pressure drop for fin-and-tube heat exchangers with rectangular winglet-type vortex generators. Applied Thermal Engineering 61: 770-783

[15] Zeeshan M, Hazarika S A, Nath S and Bhanja D 2017 Numerical investigation on the performance of fin and tube heat exchangers using rectangular vortex generators. AIP Conference Proceedings (1859): 020011

[16] Zeeshan M, Nath S, Bhanja D and Das A 2018 Numerical investigation for the optimal placements of rectangular vortex generators for improved thermal performance of finand-tube heat exchangers. Applied Thermal Engineering 136: 589-601

[17] Sinha A M, Chattopadhyay H, Iyengar A K and Biswas G 2016 Enhancement of heat transfer in a fin-tube heat exchanger using rectangular winglet type vortex generators. International Journal of Heat and Mass Transfer 101: 667-681

[18] Sharma B, Bhushan G and Sachdeva G 2017 Effect of flow structure on heat transfer in compact heat exchanger by using finite thickness winglet at acute angle. Journal of Thermal Engineering 3(2): 1149-1162

[19] Sarangi S K and Mishra D P 2017 Effect of winglet location on heat transfer of a fin and-tube heat exchanger. Applied Thermal Engineering 116: 528-540

[20] Lin C N and Jhang J Y 2002 Conjugate heat transfer and fluid flow analysis in fin-tube heat exchangers with wave-type vortex generators. Journal Enhanced Heat Transfer 9: 123-136

[21] Valentino M I, Tran L V, Ricklick M and Kapat J S 2012 A study of heat transfer augmentation for recuperative heat exchangers: comparison between three dimple geometries. Journal of Engineering for Gas Turbines and Power 134(072303): 1-9

[22] Wang C C, Chen K Y, Liaw J S and Tseng C Y 2015 An experimental study of the air-side performance of fin-andtube heat exchangers having plain, louver, and semi-dimple vortex generator configuration. International Journal of Heat and Mass Transfer 80: 281-287.

[23] Gholami A A, Wahid M A and Mohammed H A 2014 Heat transfer enhancement and pressure drop for fin-and-tube compact heat exchangers with wavy rectangular winglet-type vortex generators. International Communications in Heat and Mass Transfer 54: 132-140 
[24] Oneissi M, Habchi C, Russeil S, Bougeard D and Lemenand T 2016 Novel design of delta winglet pair vortex generator for heat transfer enhancement. International Journal of Thermal Sciences 109: 1-9

[25] Lu G and Zhou G 2016 Numerical simulation on performances of plane and curved winglet type vortex generator pairs with punched holes. International Journal of Heat and Mass Transfer 102: 679-690

[26] Song K W, Xi Z P, Su M, Wang L C, Wu X and Wang L B 2017 Effect of geometric size of curved delta winglet vortex generators and tube pitch on heat transfer characteristics of fin-tube heat exchanger. Experimental Thermal and Fluid Science 82: 8-18
[27] Zeeshan M, Nath S and Bhanja D 2017 Numerical study to predict optimal configuration of fin and tube compact heat exchanger with various tube shapes and spatial arrangements. Energy Conversion and Management 148: 737-752

[28] Zeeshan M, Nath S and Bhanja D 2019 Determination of optimum winglet height of longitudinal vortex generators for the best thermo-hydraulic performance of compact heat exchangers. Journal of Mechanical Science and Technology 33(9): 4529-4534

[29] Sun L and Zhang C L 2014 Evaluation of elliptical finnedtube heat exchanger performance using CFD and response surface methodology. International Journal of Thermal Sciences 75: 45-53 